\title{
T-DEGREES, JUMP CLASSES, AND STRONG REDUCIBILITIES
}

\author{
R. G. DOWNEY AND C. G. JOCKUSCH, JR.
}

\begin{abstract}
It is shown that there exist r.e. degrees other than $\mathbf{0}$ and $\mathbf{0}^{\prime}$ which have a greatest r.e. 1-degree. This solves an old question of Rogers and Jockusch. We call such degrees 1-topped. We show that there exist incomplete 1-topped degrees above any low r.e. degree, but also show that no nonzero low degree is 1-topped. It then

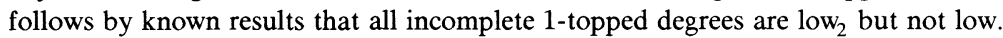
We also construct cappable nonzero 1-topped r.e. degrees and examine the relationships between 1-topped r.e. degrees and high r.e. degrees.

Finally, we give an analysis of the "local" relationships of r.e. sets under various strong reducibilities. In particular, we analyze the structure of r.e. wtt- and tt-degrees within a single r.e. T-degree. We show, for instance, that there is an r.e. degree which contains a greatest r.e. wtt-degree and a least r.e. $\mathrm{tt}$-degree yet does not consist of a single r.e. wtt-degree. This depends on a new construction of a nonzero r.e. T-degree with a least $\mathrm{tt}$-degree, which proves to have several further applications.
\end{abstract}

1. Introduction. This paper concerns itself with relationships between Turing (T-) reducibility and several other reducibilities-many-one (m-), one-one (1-), truth table (tt-), bounded truth table (btt-), and weak truth table (wtt-) - for r.e. sets and various other $\Delta_{2}^{0}$ sets. In particular in $\$ 2$ we are interested in the ordering of 1-degrees within a single r.e. T-degree. In $\$ 3$ we study connections between the (local) ordering of r.e. 1-degrees in an r.e. T-degree a and the (global) properties possessed by a within $\mathbf{R}$, the r.e. T-degrees. In $\$ 4$ we analyze (local) relationships between various reducibilities and by this we are mainly concerned with interactions of the various orderings of $r$-degrees (for various $r$ ) in a single r.e. T-degree. Thus we continue our analysis along the lines of $[\mathrm{Dg1} 1,2, \mathrm{Jo1}, 2,3$, LS, and DS]. For background information we refer to Rogers $[\mathbf{R g}]$ or Odifreddi's excellent survey [Od1].

It is well known that $\mathbf{0}^{\prime}$ and $\mathbf{0}$ are r.e.-degrees containing greatest r.e. 1-degrees. It is an old question of Rogers and Jockusch (cf. [Jo1, Problem 14-14; Rg, §4]) whether these are the only such degrees.

Let $r_{1}$ and $r_{2}$ be reducibilities. We shall say an r.e. $r_{1}$-degree $\mathbf{a}$ is $r_{2}$ - topped if there is an r.e. set $A$ of $r_{1}$-degree a such that for all r.e. sets $B$ of $r_{1}$-degree a we have $B \leqslant r_{2} A$. In this case $A$ is obviously unique up to $r_{2}$-equivalence and is called the $r_{2}$-top of a. Thus an r.e. T-degree a is tt-topped by an r.e. set $A$ if $A$ has T-degree a

Received by the editors March 21, 1986.

1980 Mathematics Subject Classification (1985 Revision). Primary 03D20, 03D25, 03 D30.

This research was carried out while the first author held a visiting position at the University of Illinois at Urbana-Champaign, and was partially supported by the NSF.

The second author was partially supported by NSF Grant DMS 83-02550. 
and for all r.e. sets $B \leqslant{ }_{\mathrm{T}} A, B \leqslant{ }_{\mathrm{tt}} A$. The question above may be rephrased in our terminology as asking if there exist nontrivial 1-topped r.e. (T-) degrees. Odifreddi [Od1, Problem 12] noted that it was even unknown whether there exist nontrivial tt-topped r.e. degrees.

In $\$ 2$ we solve these questions affirmatively by constructing a nontrivial 1-topped r.e. degree. The proof makes essential use of the fact that we are dealing with r.e. 1-degrees-or r.e. $\mathrm{tt}$-degrees-within a single r.e. degree. If we drop "r.e." and simply deal with all tt-degrees of degree a, our result then fails. In fact, Jockusch [Jo2] showed that no hyperimmune degree (and hence no nonzero degree $\leqslant \mathbf{0}^{\prime}$ ) contains a greatest $\mathrm{tt}$-degree. However, as we shall see in $\$ 4$, it is possible for an r.e. degree a to contain a least $\mathrm{tt}$-degree (amongst all sets of degree a). With this in mind we define an r.e. $r_{1}$-degree a to be $r_{2}$-bottomed if there is an r.e. set $A$ of $r_{1}$-degree a such that for all r.e. sets $B$ of $r_{1}$-degree a we have $A \leqslant r_{2} B$, and we define an a to be strongly $r_{2}$-bottomed (by $A$ ) if for all sets $B$ of $r_{1}$-degree a we have $A \leqslant_{r_{2}} B$.

In $\$ 3$ we analyze the $\mathbf{R}$-global properties possessed by 1 -topped r.e. degrees. In particular we analyze how 1-topped r.e. degrees behave under the jump operator. We show that no nonrecursive semilow r.e. set is an $\mathrm{m}$-top and no nonrecursive low r.e. set is a tt-top. From a known result of Jockusch [Jo3] it follows that all nontrivial tt-topped degrees are low $_{2}$ but not low. We also construct incomplete 1-topped r.e. degrees above each low r.e. degree. In particular, this means that there exist incomplete promptly simple 1-topped r.e. degrees (by e.g. [AJSS]). We remark that many characterizations of promptly simple degrees appear in [AJSS], and in particular promptly simple degrees are exactly the noncappable r.e. degrees (i.e. those nonzero r.e. degrees that are not part of a minimal pair of r.e. degrees). To complete $\S 3$ we show that there exist cappable 1-topped r.e. degrees, and examine the relationship between high r.e. degrees and 1-topped r.e. degrees.

In $\$ 4$ our focus shifts to the interrelationships between various reducibilities at a more "local" level (rather than the "global" aspects of §3). The main theme of this section is the structure of the tt-degrees within a single T- (or wtt-) degree. We start with a direct proof of Kobzev's [Ko3] result that there exist nonzero r.e. T-degrees with strong tt-bottoms. Our construction is much more flexible than Kobzev's indirect method (using " $\eta$-maximal semirecursive sets") and allows us to solve a question of Odifreddi [personal communication] by constructing a strong r.e. tt-bottom which is not of minimal tt-degree.

Strongly tt-bottomed r.e. degrees may or may not be (strongly) contiguous, where (following [Do2]) an r.e. degree is called contiguous (resp. strongly contiguous) if it contains a single r.e. wtt-degree (resp. single wtt-degree). These and other results enable us to show that there are

(1) low 2 r.e. degrees that are 1-topped but not tt-bottomed,

(2) strongly tt-bottomed and strongly contiguous r.e. degrees,

(3) strongly tt-bottomed and not wtt-topped r.e. degrees, and

(4) wtt-topped strongly tt-bottomed noncontiguous r.e. degrees.

Many of these results are to some extent the best possible by various results from Lachlan [La2], Cohen [Co], and Kobzev [Ko1, 2, 3]. 
Two other results from $\S 4$ are the following. First, there exists an r.e. T-degree that contains infinitely many r.e. wtt-degrees, the structure of which is a lattice with least and greatest members. Second, we generalize the Friedberg and Rogers [FR] result that hypersimple r.e. sets are not wtt-complete by showing that hypersimple r.e. sets are not wtt-cuppable. That is, if $H$ is hypersimple and r.e., and $D$ is any r.e. set with $D \oplus H \equiv{ }_{\text {wtt }} \varnothing^{\prime}$ then $D \equiv{ }_{\text {wtt }} \varnothing^{\prime}$. In particular each r.e. T-degree contains r.e. wtt-degrees that are not wtt-cuppable and no contiguous r.e. degree is wtt-cuppable. (This last result is due to Ambos-Spies, Jockusch, Soare, and Shore in [AJSS].) Finally, we show that $\mathbf{0}_{\mathrm{T}}^{\prime}$ fails to be wtt-bottomed in a very strong way. Namely, we show that if $A$ is any r.e. set with $A \leqslant{ }_{\text {wt }} B$ for all r.e. sets $B$ of T-degree $\mathbf{0}^{\prime}$ then $A \equiv{ }_{\mathrm{T}} \varnothing$.

Our notation is for the most part standard and will follow the rules that $\Phi_{e}$ and $\Gamma_{e}$ will denote T-functionals, $\hat{\Gamma}_{e}$ will denote a wtt-functional with use $\gamma_{e}$, and in general $\gamma_{e}$ will denote a partial recursive function. $W_{e}$ will denote the $e$ th r.e. set. The arguments of all computations, etc. will be bounded at any stage $s$ by $s$. We denote by $\alpha_{e}$ the $e$ th tt-condition, so that $B \leqslant{ }_{\mathrm{tt}} A$ iff there is a recursive function $f$ such that for all $x, x \in B$ iff $A \vDash \alpha_{f(x)}$. When convenient we suppose that use functions and $\operatorname{dom} \alpha_{x}$ are both monotone in $x$. We denote by $u(-)$ the use function of any computation (-). We let dtt and btt denote, respectively, disjunctive truth table and bounded truth table reducibility. Here and elsewhere we refer to Odifreddi [Od1].

Finally, most of our constructions use "tree of strategies" constructions, presented in the manner of Stob [St]. It would be helpful if the reader were familiar with this or Soare [So3 or So4]. We do abuse notation slightly by sometimes using $W_{e}$ for different roles in the same construction. However, we shall only do so when the meaning will be clear from the context.

The authors wish to thank Mike Stob for several helpful suggestions regarding this material.

2. A 1-topped r.e. degree. The goal of this section is to give the basic construction of a 1-topped r.e. degree.

(2.1) Theorem. There exists an r.e. degree $\mathbf{a} \neq \mathbf{0}, \mathbf{0}^{\prime}$ such that $\mathbf{a}$ is 1-topped.

Proof. We shall build $A=\cup_{s} A_{s}$ together with an auxiliary set $B=\cup_{s} B_{s}$ in stages, to satisfy the requirements:

$$
\begin{aligned}
& P_{e}: \bar{A} \neq W_{e}, \\
& Q_{e}: \neg\left(B \leqslant{ }_{1} A \text { via } \gamma_{e}\right), \\
& R_{e}: \Phi_{e}(A)=W_{e} \text { implies } W_{e} \leqslant_{1} A .
\end{aligned}
$$

Here, for the $Q_{e}$ we suppose $\left\{\gamma_{e}\right\}_{e \in \omega}$ is a recursive list of all 1-1 partial recursive functions, and for the $R_{e}$ we regard $\left(\Phi_{e}, W_{e}\right)$ as a list of all pairs consisting of a T-functional $\left(\Phi_{e}\right)$ and an r.e. set $\left(W_{e}\right)$. Our priority ranking here is $R_{0}, P_{0}, Q_{0}, R_{1}, \ldots$

We shall satisfy the $P_{e}$ in the usual way: we appoint followers $x$, wait till $x$ appears in $W_{e}$ and then enumerate $x$ into $A$ when this occurs. The basic idea for the satisfaction of the $Q_{e}$ requirements is also quite simple. We pick a follower $x$ 
targeted for $B$ and wait till $\gamma_{e, s}(x) \downarrow$. We then enumerate $x$ into $B$ iff $\gamma_{e, s}(x) \notin A_{s}$. If $\gamma_{e, s}(x) \notin A_{s}$ we restrain $A$ in the sense that we would not allow $\gamma_{e, s}(x)$ to be enumerated into $A$. (Strictly speaking we attempt to restrain by cancellation and use the fact that $s$ bounds all current computations.)

Our problems occur with the satisfaction of the $R_{e}$ requirements in conjunction with the $P_{i}$ and $Q_{i}$ requirements. First we need to describe the method of satisfaction of the $R_{e}$. Let $l(e, s)=\max \left\{x: \forall y<x\left(\Phi_{e, s}\left(A_{s} ; y\right)=W_{e, s}(y)\right)\right\}$.

Convention. Here we regard $\Phi_{e, s}\left(A_{s}\right)$ as controlling $W_{e, s}$ and do not allow $W_{e, s}$ to change until allowed to by $\Phi_{e, s}\left(A_{s}\right)$. Explicitly, this means that once $l(e, s)>y$ then for all $t>s, W_{e, s}(y)=W_{e, t}(y)$ unless some number $z$ enters $A_{t}-A_{s}$ with $z<u\left(\Phi_{e, s}\left(A_{s} ; y\right)\right)$. We shall adopt this convention here and for our other constructions. This convention is important since it allows us to control $W_{e, s}$ by controlling $A_{s}$.

Let $m l(e, s)$ denote $\max \{l(e, t): t<s\}$. We say a stage is e-expansionary if $l(e, s)>m l(e, s)$. For a single $R_{e}$ requirement, roughly speaking, our idea is as follows. We intend to give each $y \in \omega$ a trace $f(e, y)$ and ensure that $y \in W_{e}$ iff $f(e, y) \in A$. Thus at $e$-expansionary stages, we give each $y<l(e, s)$ not already possessing a trace, a trace $f(e, y)$ which will be a large fresh number. Now we promise that if ever $y \in W_{e, s}$ then $f(e, y) \in A_{s+1}$. (The reader should note that the convention above on $W_{e, s}$ will mean, in a sense, we will cause such $y \in W_{e, s}$ since such $y$ can enter only when we change $A$.)

By choosing followers of $P_{e}$ correctly, this will not interfere with the satisfaction of the $P_{e}$ requirements. However the combination of this $P_{e}$ with the $R_{e}$ may affect the $Q_{e}$. When $x$ enters $A$ for the sake of some $P_{j}$, this allows $A$ to change and so $W_{e}$ to change and hence perhaps force many traces into $A$, because of our commitments to $R_{k}$ for $k \leqslant e$. Now in the situation to satisfy the $Q_{e}$ we wait till $\gamma_{e, s}(x) \downarrow$, and act according to whether or not $\gamma_{e, s}(x) \in A_{s}$. It is however possible that $\gamma_{e, s}(x) \downarrow$ but $\gamma_{e, s}(x)=f(i, y)$ for some $i \leqslant e$, and $\gamma_{e, s}(x) \notin A_{s}$. Hence although we set $B_{s+1}=B_{s}$ $\cup\{x\}$, perhaps at some later stage $t, A_{t}\left[u\left(\Phi_{i, t}\left(A_{t} ; y\right)\right)\right]$ changes, allowing $y$ to enter $W_{i}$ and now forcing $f(i, y)=\gamma_{e}(x)$ into $A$. This problem is acute due to the infinitary nature of the $R_{e}$ requirements.

Our solution is to be more shrewd in our choice of stages at which to attack the $Q_{e}$. Thus we wait for a stage $s$ where $s$ is $i$-expansionary (for each "appropriate" $i \leqslant e)$ and for all $z<u\left(\Phi_{i, s}\left(A_{s} ; y\right)\right), z \in W_{i, s}$ iff $f(i, z) \in A_{s}$. We then cancel all lower priority followers and traces for $R_{k}$ and $P_{k}$ for $k>e$ and know that with priority $e$ we have satisfied $Q_{e}$ since $A\left[u\left(\Phi_{i, s}\left(A_{s} ; y\right)\right)\right]$ is now fixed.

Now, as usual, we do not know which $i \leqslant e$ are appropriate (that is, have $l(i, s) \rightarrow \infty)$ and this necessitates some sort of nested strategies procedure. We choose to use Lachlan's tree of strategies approach. This method equips $Q_{e}$ with guesses as to the action of higher priority requirements. The crucial definition is that of " $\sigma$-correct computation" which, as in (say) a high minimal pair, lies at the heart of the construction. For more on tree arguments the reader is referred to Soare [So2, and So3, Chapter XIV].

We now give the formal details of the argument. Let $T=2^{<\omega} . R_{e}$ is identified with those $\sigma \in 2^{<\omega}$ with $\ln (\sigma)=e+1$ where $\operatorname{lh}(\sigma)$ denotes the length of $\sigma$. 
Members of $2^{<\omega}$ are referred to as guesses. We let $\sigma \subseteq \tau$ denote $\sigma$ is an initial segment of $\tau$ and $\sigma \leqslant{ }_{L} \tau$ the usual left tree ordering: $\sigma \leqslant_{L} \tau$ iff $\sigma \subseteq \tau$ or $\exists \gamma\left(\gamma^{\wedge} 0 \subseteq \sigma \& \gamma^{\wedge} 1 \subseteq \tau\right)$. We remark that $\sigma \leqslant{ }_{L} \tau$ should be read as $\sigma$ is stronger than $\tau$ (for $\sigma \neq \tau$ ).

Instead of $f(e, y)$ above, we shall build guessed versions $f\left(\sigma^{\wedge} 0, y\right)$ for each $\sigma^{\wedge} 0$ with $\operatorname{lh}(\sigma)=e$. A guess $\sigma^{\wedge} 0$ is guessing that $\Phi_{e}(A)=W_{e}$ plus whatever $\sigma$ encodes. In fact $f\left(\sigma^{\wedge} 0, \cdot\right)$ will be partial recursive but have cofinite domain in the second variable for each "truly correct" guess $\sigma^{\wedge} 0$. Also dom $f$ is finite at each stage $s$. It is important to note that dom $f\left(\sigma^{\wedge} 0,-\right)$ is only extended at $\sigma^{\wedge} 0$-stages (which we define later). The key definition is

(2.2) Definition. We say a computation $\Phi_{e, s}\left(A_{s} ; x\right)=W_{e, s}(x)$ is $\sigma$-correct at stage $s$ where $\operatorname{lh}(\sigma)=e+1$ and $\sigma=\tau^{\wedge} 0$ if $l(e, s)>x$ and for all $\rho^{\wedge} 0 \leqslant{ }_{L} \sigma$ and all $z$ if

(i) $f\left(\rho^{\wedge} 0, z\right)$ is defined, and

(ii) $f\left(\rho^{\wedge} 0, z\right) \leqslant u\left(\Phi_{e, s}\left(A_{s} ; x\right)\right)$, then

(iii) $z \in W_{i, s}$ iff $f\left(\rho^{\wedge} 0, z\right) \in A_{s}$ for $i=\ln (\rho)$.

The reader should note that the above must hold for all $\rho^{\wedge} 0 \leqslant{ }_{L} \sigma$ not just $\rho^{\wedge} 0 \subseteq \sigma$. Roughly speaking, (2.2) says that $\sigma$ does not believe any computation until all higher priority $R_{i}$ action that might affect the computation has been completed.

(2.3) We define the notions $l(\sigma, s)$ and $\sigma$-stage by induction on $l h(\sigma)$ and $s$.

(i) Every stage $s$ is a $\varnothing$-stage.

(ii) If $s$ is a $\tau$-stage with $\operatorname{lh}(\tau)=e$ then we define

$$
l\left(\tau^{\wedge} 0, s\right)=\max \left\{x: \forall y<x\left(\Phi_{e, s}\left(A_{s} ; y\right)=W_{e, s}(y)\right.\right.
$$

and these computations are $\tau^{\wedge} 0$-correct at stage $\left.\left.s\right)\right\}$.

Then if $l\left(\tau^{\wedge} 0, s\right)>\max \left\{l\left(\tau^{\wedge} 0, t\right): t\right.$ is a $\tau^{\wedge} 0$-stage and $\left.t<s\right\}$, we say $s$ is a $\tau^{\wedge} 0$-stage. Otherwise $s$ is a $\tau^{\wedge} 1$-stage.

Now let $\sigma_{s}$ denote the unique guess of length $s$ such that $s$ is a $\sigma_{s}$-stage. Following the ideas of Stob [St], followers of $P_{e}$ and $Q_{e}$ are given guesses $\sigma$ with $\operatorname{lh}(\sigma)=e+1$. We shall say that $P_{e}$ requires attention at stage $s+1$ if $W_{e, s} \cap A_{s}=\varnothing$ and one of the following options holds.

(2.4) $P_{e}$ has no follower.

(2.5) $P_{e}$ has a follower $x$ with $x \in W_{e, s}$.

We say that $Q_{e}$ requires attention at stage $s+1$ if $Q_{e}$ is not currently declared satisfied, and one of the following options holds.

(2.6) $Q_{e}$ has no follower with guess $\subseteq \sigma_{s}$.

(2.7) $Q_{e}$ has a follower $x$ with guess $\sigma \subseteq \sigma_{s}$ and the following conditions are satisfied.

(i) $\gamma_{e, s}(x) \downarrow$.

(ii) For all $\tau^{\wedge} 0 \subseteq \sigma, l\left(\tau^{\wedge} 0, s\right)>\gamma_{e, s}(x)$.

At each stage $s$ we take as the requirement to require attention the one of highest priority according to the above definitions and the given scheme. 
Construction, Stage $s+1$.

Step 1 (CANCellation). For all $\tau \nless_{L} \sigma_{s}$, cancel all $f(\tau, x)$ and followers with guess $\tau$, and declare as unsatisfied any $Q_{e}$ that is currently satisfied by a number with guess $\tau$.

Step 2 (Trace Assignment). For each $\tau^{\wedge} 0 \subseteq \sigma_{s}$ and for each $x<l\left(\tau^{\wedge} 0, s\right)$ if $f\left(\tau^{\wedge} 0, x\right)$ has not as yet been defined at any stage $t \leqslant s$, find a large fresh number $y(x)>s$ and define $f\left(\tau^{\wedge} 0, x\right)=y(x)$.

Step 3 (ATtEnd $P_{e}, Q_{e}$ ). Find the requirement $R$ of highest priority to require attention. Then $R=P_{e}$ or $Q_{e}$ for some $e$.

Substep 1. First cancel all followers with guess $\tau$ and traces $f(\tau, z)$ for all $\tau$ with $\sigma \subseteq \tau$ and $\sigma \neq \tau$ where $\operatorname{lh}(\sigma)=e+1$ and $\sigma \subseteq \sigma_{s}$.

Substep 2. Adopt the appropriate case below.

Case 1. $R=P_{e}$ and (2.4) holds. Appoint a large fresh number $x$ as a follower of $P_{e}$ with guess $\sigma$ where $\sigma \subseteq \sigma_{s}$ and $\ln (\sigma)=e+1$.

Case 2. $R=P_{e}$ and (2.5) holds. Enumerate $x$ into $A_{s+1} . P_{e}$ is now met (forever). Declare those $Q_{j}$ for $j \geqslant e$ as unsatisfied.

Case 3. $R=Q_{e}$ and (2.6) holds. Appoint a large fresh number as a follower of $Q_{e}$.

Case 4. $R=Q_{e}$ and (2.7) holds. Set $B_{s+1}=B_{s} \cup\{x\}$ if $\gamma_{e, s}(x) \notin A_{s}$. Otherwise set $B_{s+1}=B_{s}$. Declare $Q_{e}$ is currently satisfied via $x$.

Step 4 (attending $R_{e}$ ). For each $\gamma^{\wedge} 0$ and $x$ with $f\left(\gamma^{\wedge} 0, x\right)$ defined and $x \in W_{e, s}$ where $e=\operatorname{lh}(\gamma)$,

(2.8) If $f\left(\gamma^{\wedge} 0, x\right) \notin A_{s}$ enumerate $f\left(\gamma^{\wedge} 0, x\right)$ into $A_{s+1}$.

Please note that we do not ask that $\gamma^{\wedge} 0 \subseteq \sigma_{s}$ here.

END OF CONSTRUCTION.

Verification. Let $\beta$ denote the leftmost path. Namely $\beta \in 2^{\omega}$ with $\beta$ defined by induction on substrings: $\varnothing \subseteq \beta$ and $\sigma \subseteq \beta$ implies $\sigma^{\wedge} 0 \subseteq \beta$ iff $\exists^{\infty_{S}}(s$ is a $\sigma^{\wedge} 0$-stage), otherwise $\sigma^{\wedge} 1 \subseteq \beta$.

We first verify that all the $P_{j}$ for $j \leqslant e$ receive attention finitely often and $Q_{j}$ for $j \leqslant e$ receive attention finitely often at $\sigma$ stages where $\sigma \subseteq \beta$ and $\ln (\sigma)=e+1$, and all are eventually met. Fix $\sigma \subseteq \beta$ with $\operatorname{lh}(\sigma)=e+1$. Let $s_{0}$ be a $\sigma$-stage such that for all $s>s_{0}$

(i) $\sigma \leqslant{ }_{L} \sigma_{s}$.

(ii) $s$ is a $\sigma$-stage implies $P_{j}$ and $Q_{j}$ for $j<e$ do not receive attention at stage $s$.

(iii) For all $z, \tau$ such that $\tau^{\wedge} 0 \leqslant{ }_{L} \sigma, \tau^{\wedge} 0 \nsubseteq \sigma$, and $f\left(\tau^{\wedge} 0, z\right)$ defined, $f\left(\tau^{\wedge} 0, z\right)$ $\in A$ iff $f\left(\tau^{\wedge} 0, z\right) \in A_{s_{0}}$. Note that (iii) is possible since $f\left(\tau^{\wedge} 0, z\right)$ are only appointed at $\tau^{\wedge} 0$-stages, and since there are only finitely many $\tau^{\wedge} 0$-stages, only finitely many $f\left(\tau^{\wedge} 0, z\right)$ are ever defined.

Now if $P_{e}$ has no follower at stage $s_{0}$ it will get one with guess $\sigma$. If $P_{e}$ already has a follower it must have guess $\tau \leqslant_{L} \sigma_{s}$. In either case such a follower is now uncancellable, and so after stage $s_{0}(2.4)$ cannot pertain to $P_{e}$. Hence $P_{e}$ can require attention at most once more after stage $s_{0}$ (namely when (2.5) holds) and thereafter is met.

We now turn to the activity of the $Q_{e}$. Let $s_{1}>s_{0}$ be a $\sigma$-stage such that also

$$
\forall s>s_{1}\left(P_{e} \text { does not receive attention at stage } s\right) \text {. }
$$


Let $s_{2}>s_{1}$ be a $\sigma$-stage. Now at stage $s_{2}+1, Q_{e}$ must have an uncancellable follower $x$ with guess $\sigma$. Certainly $Q_{e}$ is met and will never again receive attention at a $\sigma$-stage if $\gamma_{e}(x) \uparrow$. Similarly, if $\gamma_{e}(x) \downarrow$ and $\gamma_{e}(x) \in A_{s}$ with $x \notin B_{s}$ for some stage $s \geqslant s_{2}$, then $Q_{e}$ is clearly thereafter forever met. (Nothing will induce us to add $x$ to $B$.) Hence the only situation we need worry about is exactly the one discussed in the introduction, that all the mechanics of the construction were set up for. Thus let $s_{3} \geqslant s_{2}$ be the least $\sigma$-stage with $\gamma_{e, s_{3}}(x) \downarrow$ and $\gamma_{e, s_{3}}(x) \notin A_{s_{3}}$. Now, as $\sigma \subseteq \beta$ for each $\tau^{\wedge} 0 \subseteq \sigma$ we know that $l\left(\tau^{\wedge} 0, s\right) \rightarrow \infty$ for $\sigma$-stages $s$. Hence there is a $\sigma$-stage $s_{4} \geqslant s_{3}$ such that $l\left(\tau^{\wedge} 0, s_{4}\right)>\gamma_{e}(x)$ for each such $\tau$. Now at stage $s_{4}+1, Q_{e}$ will receive attention via $x$. Thus we will cancel all lower priority followers and traces $f(\rho, z)$ for all $\rho$ with $\sigma \leqslant{ }_{L} \rho$ and $\sigma \neq \rho$ (Step 3, Substep 1).

Also, since $l\left(\tau^{\wedge} 0, s\right)>\gamma_{e}(x)$ for each $\tau^{\wedge} 0 \leqslant{ }_{L} \sigma$, we know that for all $z \leqslant \gamma_{e}(x)$,

$$
z \in W_{i, s_{4}} \quad \text { iff } \quad f\left(\tau^{\wedge} 0, z\right) \in A_{s_{4}} \text { where } i=\operatorname{lh}(\tau)
$$

by definition of $l\left(\tau^{\wedge} 0, s\right)$ and $\sigma$-correctness. Thus by cancellation and choice of $s_{4}$, we see that $\forall s>s_{4}\left(l\left(\tau^{\wedge} 0, s\right)>\gamma_{e}(x)\right.$ and $\left.l(i, s)>\gamma_{e}(x)\right)$. The reader should realize here that we are explicitly appealing to our convention concerning the way numbers may enter $W_{i}$. Thus we have achieved our desired aim that $\gamma_{e}(x) \in A$ iff $\gamma_{e}(x) \in A_{s_{4}}$. Hence we now win the requirement $Q_{e}$ in Substep 2 by ensuring $x \notin B_{s_{4}+1}$ iff $\gamma_{e}(x) \in A_{s_{4}}$ and so $x \notin B$ iff $\gamma_{e}(x) \in A$.

Finally, we argue that all the $R_{e}$ are met. Let $\gamma \subseteq \beta$ with $\operatorname{lh}(\gamma)=e+1$. Suppose $\Phi_{e}(A)=W_{e}$. Since $\Phi_{e}(A)=W_{e}$ we know that the use function of any computation is bounded. Therefore $\gamma=\tau^{\wedge} 0$. If $s_{0}$ is the stage defined at the beginning of the verification, we see that no trace $f\left(\tau^{\wedge} 0, z\right)$ (for any $z$ ) defined after stage $s_{0}$ is ever cancelled. Then the activity in Step 4 (2.8) specifically ensures that $z \in W_{e}$ iff $f\left(\tau^{\wedge} 0, z\right) \in A$. As $l\left(\tau^{\wedge} 0, s\right) \rightarrow \infty$ for $\tau^{\wedge} 0$-stages, we see that for almost all $z$, $f\left(\tau^{\wedge} 0, z\right)$ is defined after stage $s_{0}$ and so $W_{e} \leqslant{ }_{1} A$.

There are several corollaries or easy modifications to the above. Using infinitary Sacks restraints with guessing as above, we may avoid cones: viz

(2.9) COROLlary. Let $\mathbf{c}$ be any nonzero $\Delta_{2}^{0}$ degree. Then there exists a 1-topped r.e. degree $\mathbf{a} \neq \mathbf{0}$ with $\mathbf{a} \neq \mathbf{c}$.

We leave the proof of (2.9) to the reader. Another corollary is motivated by the following definition: recall from [LR] that an r.e. set $A$ has USP (universal splitting property) if for all r.e. sets $B \leqslant{ }_{\mathrm{T}} A$ there is an r.e. splitting $A_{1} \sqcup A_{2}=A$ of $A$ with $A_{1} \equiv{ }_{\mathrm{T}} B$. Every nonzero r.e. degree contains an r.e. set without USP [Do1], and many r.e. degrees contain no r.e. set with USP (e.g. cf. [LR]). We obtain the following very strong USP existence theorem.

(2.10) COROLlary. There exists an r.e. set $A \not{ }_{\mathrm{T}} \varnothing, \varnothing^{\prime}$ such that for any nonsimple coinfinite r.e. set $B \leqslant{ }_{\mathrm{T}} A$ there is an r.e. splitting $A_{1} \sqcup A_{2}=A$ with $A_{1} \equiv_{1} B$. Hence for any coinfinite r.e. set $C \leqslant_{\mathrm{T}} A$ there is an r.e. splitting $A_{3} \sqcup A_{4}=A$ with $A_{3} \equiv{ }_{\mathrm{m}} C$. Any 1-topped r.e. degree contains such an r.e. set. 
Proof. Let $A$ be the 1-top of a 1-topped r.e. degree. Let $B \leqslant_{\mathrm{T}} A$ be r.e. and nonsimple. Then $B \leqslant{ }_{1} A$ and $B \oplus A \equiv{ }_{1} A$. Let $\gamma$ be a recursive permutation of $\omega$ with $\gamma(B \oplus A)=A$. Let $A_{1}=\gamma(B \oplus \varnothing)$ and $A_{2}=\gamma(\varnothing \oplus B)$. Evidently $A_{1} \sqcup A_{2}$ $=A$ and since $B$ is not simple, $B \equiv{ }_{1} B \oplus \varnothing \equiv{ }_{1} A_{1}$.

3. Classification of the 1-topped degrees. The concern of this section is to analyze the properties in $\mathbf{R}$ possessed by 1-topped degrees. The first one was found by Jockusch [Jo3] and we give it here for the reader's convenience.

(3.1) TheOrem (Jockusch [Jo3, Corollary 8(i)]). Let $\mathbf{a} \neq \mathbf{0}^{\prime}$ be an r.e. 1-topped degree. Then a is $\operatorname{low}_{2}$ (i.e. $\mathbf{a}^{\prime \prime}=\mathbf{0}^{\prime \prime}$ ).

Proof. Let $A$ be the r.e. set which is the 1-top of a. Let $G\left(\leqslant_{1} A\right)=\left\{e: W_{e} \leqslant{ }_{1} A\right\}$ and $G(\leqslant \mathbf{a})=\left\{e: W_{e} \leqslant_{\mathrm{T}} A\right\}$. Then clearly $G\left(\leqslant_{1} A\right)$ is $\Sigma_{3}^{0}$. Also $G(\leqslant \mathbf{a})$ is $\Sigma_{3}^{A}$-complete by Yates [Ya]. Now $G\left(\leqslant{ }_{1} A\right)=G(\leqslant \mathbf{a})$ and as $A$ is incomplete, this can only happen if $A$ is low $_{2}$ by Yates [Ya].

A related result we would like to mention here is also due to Jockusch in [Jo3]. Let $A$ and $B$ be r.e. We write $A \leqslant{ }_{\mathrm{pr}} B$ if $A=f^{-1}(B)$ via some primitive recursive function $f$. In [Jo3] it is shown that if $B$ is r.e., nonrecursive, and not creative then there is an r.e. set $B \equiv{ }_{\mathrm{T}} A$ such that $B \nless{ }_{\mathrm{pr}} A$.

Our next result shows that not all nonzero degrees bound nonzero 1-topped r.e. degrees. Indeed, no low r.e. degree $\neq \mathbf{0}$ is 1-topped.

(3.2) Theorem. (i) Suppose $A$ is r.e. and semilow (that is, $\left\{e: W_{e} \cap \bar{A} \neq \varnothing\right\} \leqslant \mathrm{T}$ $\left.\varnothing^{\prime}\right)$ and $E$ is r.e. and nonrecursive. Then there is an r.e. set $B \leqslant_{\mathrm{wtt}} E$ and $B \nless_{\mathrm{m}} A$.

(ii) In particular, no low nonzero r.e. degree is 1-topped.

Proof. Let $A=\cup_{s} A_{s}$ be given by some recursive enumeration. We build $B=\bigcup_{s} B_{s}$ to satisfy the requirements

$$
R_{e}: \neg\left(B \leqslant{ }_{\mathrm{m}} A \text { via } \gamma_{e}\right) .
$$

Here $\gamma_{e}$ denotes the $e$ th partial recursive function. To meet $R_{e}$ we use auxiliary sets $W_{f(e)}$ where $f$ is recursive. Let $C=\left\{e: W_{f(e)} \cap \bar{A} \neq \varnothing\right\}$. Then $C \leqslant_{\mathrm{T}} \varnothing^{\prime}$ as $A$ is semilow. Thus there is a recursive function $g(e, s)$ such that $\forall e\left(C(e)=\lim _{s} g(e, s)\right)$ by the limit lemma. By the recursion theorem we can use $g$ in the construction.

We now describe the strategy for a single $R_{e}$.

Look for $x$ and $s$ such that

(i) $x \notin B_{s}$,

(ii) $\gamma_{e, s}(x) \downarrow$, and

(iii) $E_{s+1}[x] \neq E_{s}[x]$. (We say $E$ permits on $x$ at $s$.)

Then we put $\gamma_{e, s}(x)$ into $W_{f(e), s+1}$ and search for the least $t \geqslant s$ such that $\gamma_{e}(x) \in A_{t}$ or $g(e, t)=1$. This $t$ exists, because if $\gamma_{e}(x) \notin A$ then $W_{f(e)} \cap \bar{A} \neq \varnothing$ and so $g(e, t)=1$ for all sufficiently large $t$.

If $\gamma_{e}(x) \in A_{t}$ do nothing further for $R_{e}$ since then $x \notin B$ and $\gamma_{e}(x) \in A$ and so $R_{e}$ is satisfied.

If $g(e, t)=1$ put $x$ into $B_{s+1}-B_{s}$ and do nothing further to satisfy $R_{e}$ unless $\gamma_{e}(x)$ is later enumerated into $A$. In this case, start over by searching for a new witness. 
This strategy obviously succeeds if the " $\gamma_{e}(x) \in A_{t}$ " case occurs, or if $\gamma_{e}(x) \uparrow$ for some witness, or if we put $x$ into $B$ and $\gamma_{e}(x) \notin A$. If none of these occurs then $R_{e}$ receives attention infinitely often since $E$ is nonrecursive and furthermore $W_{f(e)} \subset A$. However if we attack $R_{e}$ at stage $s$ and this attack is not successful, by assumption there exists $t \geqslant s$ with $g(e, t)=1$, and yet $W_{f(e)} \subset A$. This contradicts the assumption that $C(e)=\lim _{s} g(e, s)$.

To combine strategies we simply require that a witness $x$ for $R_{e}$ be an element of $\omega^{(e)}$. The requirements then do not interfere with each other in any way. Notice that $B \leqslant{ }_{\text {wtt }} E$ by permitting. The result follows.

A similar argument establishes

(3.3) THEOREM. (i) Suppose $A$ is r.e. and low, and $E$ is r.e. and nonrecursive. Then there is an r.e. set $B \leqslant{ }_{\mathrm{wtt}} E$ such that $B \nless_{\mathrm{tt}} A$.

(ii) Hence, no low nonzero r.e. degree is tt-topped.

Proof. In this argument, we now have that $R_{e}$ is

$$
R_{e}: \neg\left(B \leqslant{ }_{\mathrm{tt}} \text { via } \gamma_{e}\right) \text {. }
$$

This time $W_{f(e)}$ is a set of canonical indices of finite sets. We let

$$
C=\left\{e:\left(\exists u \in W_{f(e)}\right)\left[D_{u} \subset \vec{A}\right]\right\} .
$$

Now, as $A$ is low, it follows that $C \leqslant_{\mathrm{T}} \varnothing^{\prime}$ (cf. Soare [So1]) and may again be approximated by the limit lemma using $g(e, t)$.

Now attack $R_{e}$ under essentially the same conditions as before, but put $u$ into $W_{f(e), s+1}$ where $D_{u}$ is the set of $y \in \overline{A_{s}}$ mentioned in the tt-condition $\alpha_{\gamma_{e}(x)}$. Search for $t \geqslant s$ such that either $D_{u} \cap A_{t} \neq \varnothing$ or $g(e, t)=1$. If $D_{u} \cap A_{t} \neq \varnothing$ put $u^{\prime}$ into $W_{f(e), t+1}$ where $D_{u^{\prime}}$ is the set of $y \in \overline{A_{t}}$ mentioned in $\alpha_{\gamma_{e}(x)}$. Repeat this until a stage $t^{\prime} \geqslant t$ is found with $g\left(e, t^{\prime}\right)=1$. Now put $x$ into $B$ if $A_{t^{\prime}}$ does not satisfy $\alpha_{\gamma_{e}(x)}$. The remaining details are essentially the same.

It is interesting to compare this result and the existence of a 1-topped r.e. degree with the following result due to Jockusch [Jo3]. Recall from [Jo3] that if a is an r.e. degree then a class $C$ of unary functions is called a-subuniform if there is a binary function $f$ of degree $\leqslant$ a such that $C \subseteq\left\{f_{e}: e \in \omega\right\}$. (Here $f_{e}$ denotes $\lambda n(f(e, n))$.) Define an r.e. degree $\mathbf{b}$ to be 1-bounding over an r.e. degree $\mathbf{a}$ if there exists an r.e. set $B$ of degree $\mathbf{b}$ such that if $C$ is any r.e. set degree $\leqslant \mathbf{a}$, then $C \leqslant{ }_{1} B$. Finally define an r.e. degree $\mathbf{b}$ to be 1-bounding if it is 1-bounding over some lesser r.e. degree $\mathbf{a} \neq \mathbf{0}$. (By (3.3) we see no low degree is 1-bounding.) We have

(3.4) Theorem. (a) (JocKuSCH [Jo3]). If $\mathbf{a}$ and $\mathbf{b}$ are r.e. with $\mathbf{b} \leqslant \mathbf{a}$ and $\mathbf{b}<\mathbf{0}^{\prime}$ then the following three statements are equivalent:

(i) the r.e. sets of degree $\leqslant \mathbf{b}$ are $\mathbf{a}$-subuniform,

(ii) $\mathbf{b}^{\prime \prime}=\mathbf{a}^{\prime}=\mathbf{0}^{\prime \prime}$,

(iii) there is an r.e. sequence of r.e. sets which is uniformly of degree $\leqslant \mathbf{a}$ and consists exactly of the r.e. sets of degree $\leqslant \mathbf{b}$.

(b) In particular, every high r.e. degree a is 1-bounding (over any low 2 r.e. degree $\mathbf{b} \leqslant \mathbf{a})$. 
Proof. (b) Let $\mathbf{a}$ be high and $\mathbf{b} \leqslant \mathbf{a}$ be nonzero and $\operatorname{low}_{2}$. By (a)(iii) we have an r.e. sequence of r.e. sets $\left\{A_{i} A\right\}_{i \in \omega}$ which consists exactly of the r.e. sets of degree $\leqslant \mathbf{b}$ and this sequence is uniformly of degree $\leqslant \mathbf{a}$. Let $A$ be an r.e. set of degree a. Then $A \oplus \oplus_{i \in \omega} A_{i}$ is an r.e. set of degree a which 1-bounds all r.e. sets of degree $\leqslant \mathbf{b}$.

It would seem an interesting project to classify the 1-bounding r.e. degrees. It is possible that the collection of 1-bounding r.e. degrees is exactly the nonlow r.e. degrees, but this seems unlikely.

Returning to the classification of the 1-topped r.e. degrees, combining (3.1) and (3.3) we see that the nontrivial 1-topped r.e. degrees form a subset of the $\operatorname{low}_{2}-\operatorname{low}_{1}$ r.e. degrees. On the other hand, we have

(3.5) TheOREM. Let $\mathbf{c}$ be a low r.e. degree. Then there exists an incomplete 1-topped r.e. degree $\mathbf{a} \geqslant \mathbf{c}$.

Proof. Rather than give complete details, we explain how the proof of the existence of a nontrivial 1-topped degree (2.1) can be modified to give the result at hand. Thus we assume the reader is completely familiar with the proof of Theorem 2.1 .

Let $C$ be an r.e. set of the given low degree c. We construct an auxiliary r.e. set $B$ to satisfy the same requirements as in (2.1) except that the requirements $P_{e}$ are dropped and the requirements $R_{e}$ are modified by replacing $A$ by $A \oplus C$. Thus our requirements are the following:

$Q_{e}: \neg\left(B \leqslant{ }_{1} A\right.$ via $\left.\gamma_{e}\right)$.

$R_{e}: \Phi_{e}(A \oplus C)=W_{e}$ implies $W_{e} \leqslant{ }_{1} A$.

Note that these requirements imply that $C \leqslant{ }_{1} A$, by applying $R_{e}$ to an $e$ chosen so that $\Phi_{e}(A \oplus C)=W_{e}=C$. Thus the theorem holds for $\mathbf{a}=\operatorname{deg}(A)=$ $\operatorname{deg}(A \oplus C)$ if the requirements are all satisfied.

The basic strategies for satisfying $Q_{e}$ and $R_{e}$ are as in (2.1). These requirements conflict as in (2.1), but the added difficulty here is that in satisfying $Q_{e}$ we may not use $A$ alone to control $W_{i}$ for the "appropriate" $i \leqslant e$, but rather we must use $A \oplus C$. Of course this is a new kind of obstacle because $C$ is not under our control. This obstacle is overcome by using the "Robinson technique" (see [So4, Chapter XI, Theorem 3.2]) to avoid excessive reliance on $C$-false computations. The Robinson technique is combined with our guessing strategy so that, for each string $\sigma$ of length $e+1$, there is an auxiliary set $W_{h(\sigma)}$ used to meet $Q_{e}$, where $h$ is a recursive function. (Actually we may use several "versions" of $W_{h(\sigma)}$.) Let

$$
E=\left\{\sigma:\left(\exists u \in W_{h(\sigma)}\right)\left[D_{u} \subset \bar{C}\right]\right\} .
$$

Then $E \leqslant{ }_{\mathrm{T}} C^{\prime} \leqslant{ }_{\mathrm{T}} \varnothing^{\prime}$, so by the limit lemma there is a recursive function $g(\sigma, s)$ with $\lim _{s} g(\sigma, s)=E(\sigma)$ for all $\sigma$. By the recursion theorem we may assume that $h$ and $g$ are known during the construction. When $Q_{e}$ requires attention via a follower $x$ with guess $\sigma$ at stage $s$, there will be at most one pair $\langle i, y\rangle$ such that $i \leqslant e$, $y \notin W_{i, s}$, and $f(y, \tau)=\gamma_{e, s}(x)$, where $\tau^{\wedge}\langle 0\rangle \subset \sigma$ and $\tau$ has length $i$. As in (2.1), we would like to put $x$ into $B$ to meet $R_{e}$, but our attempt will be ruined if $y$ later 
enters $W_{i}$. We thus require (if such a pair exists) that $\Phi_{i, s}\left(A_{s} \oplus C_{s} ; y\right)=0$ and enumerate $u$ into $W_{h(\sigma)}$, where $D_{u}$ is the set of numbers whose nonmembership in $C_{s}$ is used in this computation. We then find the least $t \geqslant s$ such that either $g(\sigma, t)=1$ or $D_{u} \cap C_{t} \neq \varnothing$. If $g(\sigma, t)=1$ and $\gamma_{e, s}(x) \notin A_{t}$, we put $x$ into $B$, and otherwise we do nothing. If we later realize that $D_{u} \cap C \neq \varnothing$ (having put $x$ into $B$ as above), we cancel $x$ and thus may choose a new follower for $R_{e}$ with guess $\sigma$, but we continue to use the same version of $W_{h(\sigma)}$. However, at any stage $t$ with $\sigma_{t} \leqslant{ }_{L} \sigma$ and $\sigma_{t} \nsubseteq \sigma$, we start over with a new version of $W_{h(\sigma)}$.

We now give some further technical details of the argument, although these are quite straightforward, and the reader may prefer to work them out for himself.

The definitions prior to the construction are the same as in Theorem 2.1, except that all references to the $P_{e}$ 's are dropped, and $A_{s}$ is replaced by $A_{s} \oplus C_{s}$ whenever $A_{s}$ occurs as an oracle. We will also have auxiliary functions $g$ and $h$, where $W_{h(\sigma, k)}$ should be thought of as the $(k+1)$ st version of $W_{h(\sigma)}$, and

$$
\lim _{s} g(\sigma, k, s)=E(\sigma, k),
$$

where

$$
E=\left\{\langle\sigma, k\rangle:\left(\exists u \in W_{h(\sigma, k)}\right)\left[D_{u} \cap C=\varnothing\right]\right\} .
$$

The construction is the same with the following exceptions. In Step 1 (cancellation), also cancel all followers $x$ of any $Q_{e}$ such that $x \in B_{s}$ and $\gamma_{e, s}(x) \in A_{s}$. Declare all such $Q_{e}$ 's to be unsatisfied. In Step 2 (trace assignment), require that $f\left(\tau^{\wedge} 0, x\right) \in \omega^{\langle\tau, x\rangle}$, so that the pair $\langle i, y\rangle$ in our previous discussion will be unique if it exists. Cases 1 and 2 of Substep 2 of Step 3 are omitted. The main change is in Case 4 of Substep 2 of Step 3. As before in this case, if $\gamma_{e}(x) \in A_{s}$, let $B_{s+1}=B_{s}$, and declare $Q_{e}$ to be satisfied. Assume now that $\gamma_{e}(x) \notin A_{s}$, and ask if there exist $y$ and $\tau$ such that $\tau^{\wedge} 0 \subset \sigma_{s}$, and $f(y, \tau)=\gamma_{e}(x)$. If no such $y, \tau$ exist, let $B_{s+1}=B_{s}$ $\cup\{x\}$, and declare $Q_{e}$ to be satisfied. Assume now that such $y$ and $\tau$ exist. Then $y$ and $\tau$ are uniquely determined, since $\gamma_{e}(x) \in \omega^{\langle y, \tau\rangle}$. By (2.7)(ii), $l\left(\tau^{\wedge} 0, s\right)>\gamma_{e}(x)$ $=f(y, \tau)>y$. (We have $f(y, \tau)>y$ by the way we choose to define $f$.) Thus $\Phi_{i, s}\left(A_{s} \oplus C_{s} ; y\right)=W_{i, s}(y)$ by a $\tau^{\wedge} 0$-correct computation. Let $D_{u}$ be the set of numbers whose nonmembership in $C_{s}$ is used in this computation, and let

$$
k=\left|\left\{t<s: \sigma_{t} \leqslant{ }_{L} \sigma_{s} \& \sigma_{t} \not \subset \sigma_{s}\right\}\right| .
$$

Enumerate $u$ into $W_{h(\sigma, k)}$, and let $t \geqslant s$ be minimal so that $g(\sigma, k, t)=1$ or $D_{u} \cap C_{t} \neq \varnothing$. If $g(\sigma, k, t)=1$, let $B_{s+1}=B_{s} \cup\{x\}$ and declare $Q_{e}$ to be satisfied. Otherwise (in which case $D_{u} \cap C_{t} \neq \varnothing$ ), let $B_{s+1}=B_{s}$. In this final case, do not declare $Q_{e}$ to be satisfied or even regard $Q_{e}$ as having received attention, and pass to the least $e^{\prime}>e$ (if any) such that $Q_{e^{\prime}}$ requires attention at $s$, and treat it similarly. Repeat this process until either some $Q_{\hat{e}}$ receives attention at $s$, or no $e^{\prime}$ remains such that $Q_{e^{\prime}}$ requires attention at $s$ and has not been considered. This completes the description of the construction.

The verification that the $R_{e}$ 's are met is as before. To treat $Q_{e}$, let $\sigma$ be the initial segment of the leftmost path of length $e+1$. We must show that $Q_{e}$ receives attention only finitely often at $\sigma$ stages and is met. (However, it may happen that $Q_{e}$ 
requires attention infinitely often at $\sigma$ stages.) Let $k=\left|\left\{t: \sigma_{t} \leqslant{ }_{L} \sigma \& \sigma_{t} \nsubseteq \sigma\right\}\right|$. There are two cases.

Case $1 .\langle\sigma, k\rangle \in E$, so there exists $u$ in $W_{h(\sigma, k)}$ with $D_{u} \subset \bar{C}$. Let $s$ be the stage at which we added $u$ to $W_{h(\sigma, k)}$. Then $s$ is a $\sigma$-stage at which $Q_{e}$ receives attention via some existing follower $x$. It is easy to see as in Theorem 2.1 that $x$ witnesses the satisfaction of $Q_{e}$, and $Q_{e}$ never again requires attention at a $\sigma$-stage.

Case 2. $\langle\sigma, k\rangle \notin E$. Pick $s_{5}$ so large that $\sigma \leqslant{ }_{L} \sigma_{s}$ for all $s \geqslant s_{5}$, no $Q_{i}$ for $i<e$ receives attention at any $\sigma$-stage after $s_{5}$, and $g(\sigma, k, t)=0$ for all $t \geqslant s_{5}$. Then by construction, $Q_{e}$ is never regarded as receiving attention at any $\sigma$-stage after $s_{5}$. It remains only to show that $Q_{e}$ is met. Let $x$ be a follower of $Q_{e}$ which exists at some $\sigma$-stage $s>s_{5}$. If $\gamma_{e, s}(x) \uparrow$, then $\gamma_{e, s}(x)$ is obviously met. Suppose that $\gamma_{e, s}(x) \downarrow$.

Case 2A. $\gamma_{e, s}(x) \in A$. Then we are done unless $x \in B$. If $x \in B$, then $x$ will be cancelled and replaced by a new follower appointed after $s_{5}$. Hence we may assume without loss of generality that $x$ is appointed after stage $s_{5}$, so $x \notin B$. Thus (still assuming that $\left.\gamma_{e, s}(x) \in A\right), Q_{e}$ is met.

Case 2B. $\gamma_{e, s}(x) \notin A$. Assume for a contradiction that $Q_{e}$ is not met, so in particular $x \notin A$. Since $\gamma_{e}(x) \notin A$ and $x$ exists after stage $s_{5}, x$ is never cancelled. Thus $Q_{e}$ never has a follower other than $x$ at any $\sigma$-stage after $x$ is appointed. If $Q_{e}$ is declared satisfied at any $\sigma$-stage after $x$ is appointed, it is easily seen as in the proof of Theorem 2.1 that $Q_{e}$ is met. Thus this never happens. Hence we may see as in the proof of Theorem 2.1 that $Q_{e}$ requires attention at all sufficiently large $\sigma$-stages. Since this attention does not lead to $Q_{e}$ 's being declared satisfied at a large $\sigma$-stage, there must be a fixed pair $\langle i, y\rangle$ such that $\Phi_{i}(A \oplus C ; y)=0$ and yet, for all sufficiently large $\sigma$-stages $s, \Phi_{i, s}\left(A_{s} \oplus C_{s} ; y\right)=0$ via a computation which is $C$-incorrect. This contradiction shows that $Q_{e}$ is met and concludes our sketch of the proof of Theorem 3.5 .

Using this result we can establish several existence theorems for 1-topped r.e. degrees in several other degree classes. For example, recall that an r.e. degree a is low cuppable (cf. [AJSS]) if there is an r.e. degree $\mathbf{c}$ such that $\mathbf{c}$ is low and $\mathbf{c} \cup \mathbf{a}=\mathbf{0}^{\prime}$, and an r.e. degree a is contiguous (cf. [LS, Do2]) if it consists of a single r.e. wtt-degree. We have

(3.6) Corollary. Let $\mathbf{c}$ be any low r.e. degree. Then there exists an incomplete 1-topped low cuppable degree $\mathbf{a} \geqslant \mathbf{c}$ such that for all $\mathbf{b} \geqslant \mathbf{a}, \mathbf{b}$ is not contiguous.

Proof. Let $K$ denote a creative set. Let $C$ be an r.e. set of degree $\mathbf{c}$ with $\mathbf{c}$ low. Apply Robinson's [Ro] (cf. Soare [So4, Chapter XI, Theorem 3.2]) splitting theorem to find r.e. sets $K_{1}, K_{2}$ with $K_{1} \sqcup K_{2}=K$ an r.e. splitting of $K$ and $K_{1} \oplus C$, $K_{2} \oplus C$ both low. Let $D=K_{1} \oplus C$. Apply (3.4) to $D$ to obtain an r.e. set $A$ such that $A$ is the 1-top of a degree a $\geqslant T-\operatorname{deg}(D)$. Clearly a is low cuppable by $K_{2}$. Suppose that $Q$ is an r.e. set of contiguous degree with $A \leqslant{ }_{\mathrm{T}} Q$. Then $K_{1} \leqslant{ }_{\text {wtt }} Q$ and hence $K_{2} \oplus Q \equiv{ }_{\text {wtt }} K$. Thus $Q$ is wtt-cuppable. This contradicts the established result of [AJSS] that no contiguous r.e. set is wtt-cuppable. (See $\S 4$ for an extension of this result.) 
To complete the picture suggested by (3.5) we point out that 1-topped r.e. degrees may be cappable. Recall that $\mathbf{a}$ is cappable if there exists an r.e. degree $\mathbf{b} \neq \mathbf{0}$ with $\mathbf{a} \cap \mathbf{b}=\mathbf{0}$. In [AJSS] it is shown that every r.e. degree is either cappable or low cuppable (but not both). We have

(3.7) TheOREM. There exists an r.e. degree $\mathbf{a} \neq \mathbf{0}$ such that $\mathbf{a}$ is 1-topped and cappable.

Proof. We build $A=\cup_{s} A_{s}$ and $B=\cup_{s} B_{s}$ to satisfy

$$
\begin{aligned}
& P_{2 e}: \bar{A} \neq W_{e}, \quad P_{2 e+1}: \bar{B} \neq W_{e}, \\
& N_{e}: \Phi_{e}(A)=\Phi_{e}(B)=f \text { and } f \text { total implies } f \text { recursive, } \\
& R_{e}: \Phi_{e}(A)=W_{e} \text { implies } W_{e} \leqslant{ }_{1} A .
\end{aligned}
$$

We again use a tree of strategies $2^{<\omega} . N_{e}$ is associated with those $\sigma \in 2^{<\omega}$ with $\operatorname{lh}(\sigma)=2 e+2$ and the $R_{e}$ with those $\sigma$ of length $2 e+1$. In this construction we define restraints $r(\tau, s)$ for those $\tau$ of even length $\geqslant 2$. At stage $s$, we automatically define $R(\sigma, s)$ via

$$
R(\sigma, s)=\max \left\{r(\tau, s): \tau \leqslant{ }_{L} \sigma\right\} .
$$

This is well defined since we initialize all $r(\tau, 0)=-1$, and only reset those $r(\sigma, s)$ for $\sigma \subset \sigma_{s}$, where as usual $\sigma_{s}$ is the unique path of length $s$ with $s$ a $\sigma_{s}$-stage.

The reader should note that in this construction the important guessing is the minimal pair guessing. This forces us to only enumerate $f\left(\tau^{\wedge} 0, s\right)$ into $A$ for the sake of $R_{e}$ (where $e=\operatorname{lh}(\tau)$ ) at $\tau$-stages. This is the gist of the next series of definitions.

Definition. We define the notions $\sigma$-stage, $l(\sigma, s), r(\sigma, s)$, and $\sigma$-correctness simultaneously by induction on $\operatorname{lh}(\sigma)$.

(i) Every stage $s$ is a $\varnothing$-stage and $r(\varnothing, s)=-1$.

(ii) If $s$ is a $\tau$-stage and $\operatorname{lh}(\tau)=2 e$, define

$$
\begin{aligned}
l\left(\tau^{\wedge} 0, s\right)=\max \{x: \forall y< & x\left(\Phi_{e, s}\left(A_{s} ; y\right)=W_{e, s}(y)\right. \\
& \text { and this computation is } \left.\left.\tau^{\wedge} 0 \text {-correct }\right)\right\} .
\end{aligned}
$$

Here a computation $\Phi_{e, s}\left(A_{s} ; y\right)=W_{e, s}(y)$ is $\tau^{\wedge} 0$-correct if, for all $z$ with $f\left(\gamma^{\wedge} 0, z\right)$ defined for some $\gamma^{\wedge} 0 \subset \tau^{\wedge} 0$ we have that

$$
\begin{aligned}
R(\gamma, z) & <f\left(\gamma^{\wedge} 0, z\right)<u\left(\Phi_{e, s}\left(A_{s} ; y\right)\right) \\
& \Rightarrow z \in W_{i, s} \quad \text { iff } f\left(\gamma^{\wedge} 0, z\right) \in A_{s} \text { where } 2 i+2=\operatorname{lh}\left(\gamma^{\wedge} 0\right)
\end{aligned}
$$

Now if

$$
l\left(\tau^{\wedge} 0, s\right)>\max \left\{l\left(\tau^{\wedge} 0, t\right): t \text { is a } \tau \text {-stage and } t<s\right\}
$$

we say that $s$ is a $\tau^{\wedge} 0$-stage. Otherwise $s$ is a $\tau^{\wedge} 1$-stage. Set $r\left(\tau^{\wedge} i, s\right)=-1$.

(iii) If $s$ is a $\tau$-stage and $\ln (\tau)=2 e+1$ then let

$$
\begin{aligned}
l\left(\tau^{\wedge} 0, s\right)=\max \{x: \forall y< & x\left(\Phi_{e, s}\left(A_{s} ; y\right)=\Phi_{e, s}\left(B_{s} ; y\right)\right. \text { and } \\
& \text { these computations are } \left.\left.\tau^{\wedge} 0 \text {-correct }\right)\right\} .
\end{aligned}
$$


Here $\tau^{\wedge} 0$-correctness is defined as in (ii) mutatis mutandis. Then if

$$
l\left(\tau^{\wedge} 0, s\right)>\max \left\{l\left(\tau^{\wedge} 0, t\right): t \text { is a } \tau^{\wedge} 0 \text {-stage and } t<s\right\}
$$

declare $s$ to be a $\tau^{\wedge} 0$-stage, and set $r\left(\tau^{\wedge} 0, s\right)=-1$ and $r\left(\tau^{\wedge} 1, s\right)=s$. If $s$ is not a $\tau^{\wedge} 0$-stage declare $s$ as a $\tau^{\wedge} 1$-stage and define

$$
r\left(\tau^{\wedge} 1, s\right)=\max \left\{t: t \text { is a } \tau^{\wedge} 0 \text {-stage and } t<s\right\} .
$$

We say $P_{2 e}$ requires attention at stage $s+1$ if $A_{s} \cap W_{e, s}=\varnothing$ and for all followers $x$ of $P_{2 e}$ with guess $\subset \sigma_{s}, x \leqslant R(\sigma, s)$.

We say a number $x$ requires attention if either

(a) (i) $x$ is a follower of $P_{e}$ for some $e$, and

(ii) $x$ has guess $\tau \subset \sigma_{s}$ for some $\tau$, and

(iii) $x>R(\tau, s)$, and

(iv) $x \in W_{e, s}$, or

(b) (i) $x=f\left(\tau^{\wedge} 0, y\right)$ for some $\tau \subset \sigma_{s}$, and

(ii) $y \in W_{i, s}$ and $f\left(\tau^{\wedge} 0, y\right) \notin A_{s}$.

Construction, Stage $s+1$.

Step 1. Cancel all $f(\tau, z)$ and $r(\tau, s)$ and followers with guess $\tau$ for $\tau{ }_{L} \sigma_{s}$.

Step 2. For each $\tau^{\wedge} 0 \subset \sigma_{s}$ and $x<l\left(\tau^{\wedge} 0, s\right)$ for $\operatorname{lh}(\tau)$ even and $\geqslant 2$ if $f\left(\tau^{\wedge} 0, x\right)$ is undefined for all stages $t \leqslant s$, find a large fresh number $y(x)$ and set $f\left(\tau^{\wedge} 0, x\right)=$ $y(x)$.

Step 3. Find the least $i$ such that $P_{i}$ requires attention and as above we may suppose $i=2 e$. Appoint a large fresh number as a follower with guess $\sigma$. Cancel all $f(\tau, z)$ and $r(\tau, s)$ currently defined for $\tau{ }_{L} \sigma$.

Step 4. Now find the least number $x$ (if any) such that $x$ requires attention. If $x$ is a follower targeted for $B$, set $B_{s+1}=B_{s} \cup\{x\} \& A_{s+1}=A_{s}$. Otherwise $x$ is a follower or a trace targeted for $A$. In this case set $B_{s+1}=B_{s}$ and $A_{s+1}=A_{s} \cup\{x\}$. Notice only one number is enumerated into $A \oplus B$ at this stage, so the $f\left(\tau^{\wedge} 0, z\right)$ get into $A$ "slowly".

End of Construction.

We now sketch the verification as most of it is a fairly standard minimal pair type one or similar to (2.1). Let $\beta$ denote the leftmost path. By the same argument as (2.1), once we see that $\lim _{s} R\left(\tau^{\wedge} 0, s\right)=R\left(\tau^{\wedge} 0\right)$ exists for all $\tau^{\wedge} 0 \leqslant{ }_{L} \sigma \subset \beta$ where $\operatorname{lh}(\sigma)=2 e+1$, we see that $R_{e}$ is met since almost all $f\left(\sigma^{\wedge} 0, z\right)$ are free to enter in Step 4. Hence $\Phi_{e}(A)=W_{e}$ implies $W_{e} \leqslant{ }_{1} A$.

It is evident that for $\sigma \subset \beta, \lim _{s} R(\sigma, s)=R(\sigma)$ exists: Briefly, for $\gamma \leqslant{ }_{L} \sigma$ the first possibility is that $\gamma=\tau^{\wedge} 1$ and $\operatorname{lh}(\tau)=2 j$ for some $j$, and so there are only finitely many stages where

$$
l\left(\tau^{\wedge} 0, s\right)>\max \left\{l\left(\tau^{\wedge} 0, t\right): t \text { is a } \tau^{\wedge} 0 \text {-stage and } t<s\right\} .
$$

Hence $\lim _{s} r\left(\tau^{\wedge} 1, s\right)=r\left(\tau^{\wedge} 1\right)$ exists in this case. (Namely $r\left(\tau^{\wedge} 1\right)=\max \{t: t$ is a $\tau^{\wedge} 0$-stage $\}$.) The other possibility for $\gamma \leqslant{ }_{L} \sigma$ is that $\gamma \neq \tau^{\wedge} 1$ for any $\tau$ with $\operatorname{lh}(\tau)$ even and hence $r(\tau, s)=-1$ for all $s$. This means that $\lim _{s} R(\sigma, s)=R(\sigma)$ exists since there are only finitely many $\tau^{\wedge} 1$-stages with $\tau^{\wedge} 1 \leqslant_{L} \sigma$ but $\tau^{\wedge} 1 \not \subset \beta$. (The above is all fairly standard.) 
In view of this it is clear that all the $P_{e}$ are met since eventually $P_{e}$ must get a follower $x$ with guess $\sigma \subset \beta$ where $x>R(\sigma)$ and this follower will succeed.

It remains therefore to observe that all the $N_{e}$ are met. Let $\sigma \subset \beta$ with $\operatorname{lh}(\sigma)=2 e$ +1 . Let $s_{0}$ be a stage after which $\sigma \leqslant{ }_{L} \sigma_{s}$ and $r\left(\tau, s_{0}\right)=r(\tau)$ for all $\tau \leqslant{ }_{L} \sigma$ and all the $P_{j}$ activity for $j<e$ has ceased and all $f\left(\gamma^{\wedge} 0, z\right) \in A_{s_{0}}$ if $f\left(\gamma^{\wedge} 0, z\right) \in A$ for all $\gamma^{\wedge} 0 \leqslant{ }_{L} \sigma$ with $\gamma^{\wedge} 0 \not \subset \sigma$. Let $x$ be given. Find a $\sigma$-stage $s>s_{0}$ where $l(\sigma, s)>x$. Now at most one number not restrained at $s$ may enter $A \oplus B$ between this and the next $\sigma$-stage, as in the construction of a high minimal pair. We refer to, e.g., [So2 or So4, Chapter XIV] for further details.

The above result is the simplest of a series of such results. For example we can easily modify the argument above to show

(3.8) Corollary. There exists a minimal pair $\mathbf{a}, \mathbf{b}$ of 1-topped r.e. degrees with $\mathbf{a} \cup \mathbf{b}$ also 1-topped.

Proof. Left to reader.

(Other results would include embeddings (by 1-topped r.e. degrees) of boolean algebras, etc.)

To conclude this section we give some further limitations on the 1-topped degrees. One we should mention is

(3.9) THEOREM (LERMAN AND REMmeL [LR]). The r.e. degrees without wtt-tops form a dense subcollection of the r.e. degrees.

Our last result for this section is a partial answer arising from an attempt to connect the 1-topped degrees with another jump class. We do not know whether every high r.e. degree bounds a nonzero 1-topped r.e. degree. Our partial solution to this question is:

(3.10) THEOREM. There exists an r.e. set $A$ of high degree such that for all r.e. sets $B$ with $\varnothing<{ }_{\mathrm{T}} B \leqslant{ }_{\mathrm{wtt}} A$, wtt- $\operatorname{deg}(B)$ is not tt-topped.

Proof. Let $D=\bigcup_{e} D^{(e)}$ be a piecewise recursive set such that $D^{(e)}=\omega^{(e)}$ if $\operatorname{card}\left(W_{e}\right)=\infty$ and $D_{e}$ is a finite initial segment of $\omega^{(e)}$ otherwise. We recall that an r.e. set $Q \subset D$ with $Q^{(e)}={ }^{*} D^{(e)}$ for all $e$ is called a thick subset of $D$. As in [So2] if $Q$ is a thick subset of $D$ then $Q$ is high. Thus we construct a subset $A=\cup_{s} A_{s}$ of $D$ and a collection $C_{e}=\cup_{s} C_{e, s}$ of auxiliary r.e. sets to satisfy

$$
\begin{aligned}
& P_{e}: A^{(e)}={ }^{*} D^{(e)}, \\
& R_{e, i}: \text { If } \hat{\Phi}_{e}(A)=W_{e} \text { then } W_{e} \text { is recursive or, } \\
& \quad C_{e} \leqslant{ }_{\mathrm{wtt}} W_{e} \text { and } \neg\left(C_{e} \leqslant \mathrm{tt} W_{e} \text { via } \gamma_{i}\right) .
\end{aligned}
$$

Here $\left(\hat{\Phi}_{e}, W_{e}\right)$ is a standard enumeration of pairs of wtt-functionals $\hat{\Phi}_{e}$ with use $\phi_{e}$ and r.e. sets $W_{e}$. As usual we consider $\Phi_{e}(A)$ as controlling $W_{e}$, and $\hat{\Phi}_{e, s}\left(A_{s} ; x\right) \downarrow$ means $\Phi_{e, s}\left(A_{s} ; x\right) \downarrow$ and has use $\leqslant \phi_{e, s}(x)$ where $\phi_{e, s}(x) \downarrow$. Associated with the $R_{e, i}$ will be a collection of restraints $r(\tau, s)$ for $\tau \in 2^{<\omega}$ with $\operatorname{lh}(\tau)=\langle e, i\rangle+1$. 
(3.11) Definition. Define $\sigma$-correct, $l(\sigma, s)$, and $\sigma$-stage by induction on $\operatorname{lh}(\sigma)$ as follows:

(i) Every stage $s$ is a $\varnothing$-stage and all computations are $\varnothing$-correct.

(ii) If $s$ is a $\tau$-stage where $\operatorname{lh}(\tau)=e$ then if

$$
\operatorname{card}\left(D_{s+1}^{(e)}\right)>\max \left\{\operatorname{card}\left(D_{t}^{(e)}\right): t \text { is a } \tau \text {-stage with } t<s\right\}
$$

then $s$ is a $\tau^{\wedge} 0$-stage. Otherwise $s$ is a $\tau^{\wedge} 1$-stage. Now a computation $\hat{\Phi}_{e, s}\left(A_{s} ; x\right) \downarrow$ is $\tau^{\wedge} i$-correct for $i=0,1$ if for all $\rho^{\wedge} 0 \subset \tau^{\wedge} i$ where $\operatorname{lh}(\rho)=j$ if $r\left(\rho^{\wedge} 0, s\right)<z \leqslant$ $\gamma_{e, s}(x)$ and $z \in \omega^{(j)}$ then $z \in A_{s}$. Let $l\left(\tau^{\wedge} i, s\right)$ denote $\max \left\{x: \forall y<x\left(\hat{\Phi}_{e, s}\left(A_{s} ; y\right)\right.\right.$ $=W_{e, s}(y)$ and the computation is $\tau^{\wedge} i$-correct! $\}$.

As usual $\sigma_{s}$ denotes the unique string of length $s$ with $s$ a $\sigma_{s}$-stage. Recall $\alpha_{a}$ denotes the $a$ th tt-condition.

(3.12) Definition. We say $R_{e, i}$ requires attention at stage $s+1$ if one of the following options hold:

(3.13) For some follower $z$ of $R_{e, i}$ we have

(i) $z$ is active and has guess $\tau \leqslant{ }_{L} \sigma_{s}$, and

(ii) if we set

$$
l(e, s)=\max \left\{x: \forall y<x\left(\hat{\Phi}_{e, s}\left(A_{s} ; y\right)=W_{e, s}(y)\right)\right\},
$$

then

$$
l(e, s)>\max \{l(\tau, t): t \text { is a } \tau \text {-stage and } t<s\}
$$

and

(iii) $W_{e, s}[z] \neq W_{e, t}[z]$ where $t$ is the last $\tau$-stage less than $s$, that is

$$
t=\max \left\{t^{\prime}: t^{\prime}<s \text { and } t^{\prime} \text { is a } \tau \text {-stage }\right\} .
$$

(3.14)(a) For all followers $z$ of $R_{e, i}$ if $z$ has guess $\tau \subset \sigma_{s}$ then $z$ is active, and

(b) there does not exist a number $z$ such that

(i) $\gamma_{i, s}(z) \downarrow$ and $z \in C_{e, s}$ iff $\neg W_{e, s} \vDash \alpha_{\gamma_{i}(z)}$.

(ii) For some $\rho \leqslant{ }_{L} \tau$ with $\operatorname{lh}(\rho)=\operatorname{lh}(\tau)$ we have $r(\rho, s)>N$ and $l(e, s)>N$, where

(ii)(a) $N=\max \left\{\phi_{e}(y): y \leqslant M\right\}$, and

(ii)(b) $M=\max \left\{p: p \in \operatorname{dom} \alpha_{\gamma_{i}(z)}\right\}$.

(3.15) $R_{e}$ has an inactive follower $z$ with guess $\tau \subset \sigma_{s}, \gamma_{i, s}(z) \downarrow$ and $l(\tau, s)>$ $\max \left\{z, z^{\prime}\right\}$ where $z^{\prime}=\max \left\{p: p \in \operatorname{dom} \alpha_{\gamma_{i}(z)}\right\}$.

Construction, Stage $s+1$.

Step 1. Cancel all followers and restraints with guesses $\tau \nless{ }_{L} \sigma_{s}$.

Step 2. For each $\langle e, i\rangle\langle s$, adopt the first case below which holds.

Case 1 (SATISFYING $R_{e, i}$ ). (3.13) holds; set $r(\tau, s+1)=s+1$. Set $C_{e, s+1}=C_{e, s}$ $\cup\{z\}$ if $\neg W_{e, s} \vDash \alpha_{\gamma_{i}(z)}$. Declare $z$ to be no longer a follower. Cancel all followers with guess $\rho$ for $\tau \leqslant{ }_{L} \rho$ and $\operatorname{lh}(\tau)=\operatorname{lh}(\rho)$.

Case 2 (Follower ASSIGNMENT). (3.14) holds: appoint a large fresh number as an inactive follower of $R_{e, i}$ with guess $\sigma$ where $\sigma \subset \sigma_{s}$ and $\operatorname{lh}(\sigma)=\langle e, i\rangle+1$. 
Case 3 (Activation). (3.15) holds; declare $z$ as active.

Step 3. Finally for all $e$ and $x \in D_{s+1}^{(e)}$ if $x \notin A_{s}$ enumerate $x \in A_{s+1}$ provided that $x \geqslant r(\tau, s+1)$ for all $\tau \leqslant{ }_{L} \sigma$ where $\sigma \subset \sigma_{s}$ and $\operatorname{lh}(\sigma)=e+1$.

END OF CONSTRUCTION.

Let $\beta$ denote the leftmost path. We verify by simultaneous induction that for $\sigma \leqslant{ }_{L} \beta$ that $\lim _{s} r(\sigma, s)=r(\sigma)$ exists, the $R_{e, i}$ are met and all the $P_{e}$ are met. Thus let $\sigma$ be given, with $\operatorname{lh}(\sigma)=e+1$ and let $s_{0}$ be a stage such that for all $s>s_{0}$

(i) $\sigma \leqslant{ }_{L} \sigma_{s}$, and

(ii) $r(\tau, s)=r\left(\tau, s_{0}\right)$ for all $\tau \neq \sigma$ and $\tau \subset \sigma$.

We easily see that if $\rho^{\wedge} 1 \subset \sigma$ then $D_{s_{0}}^{(j)}=D^{(j)}$ where $j=\operatorname{lh}(\rho)$. Thus we may also suppose that

(iii) $A_{s_{0}}^{(j)}=A^{(j)}$ for all $\rho^{\wedge} 1 \subset \sigma$ with $j=\operatorname{lh}(\rho)$.

Finally we may also suppose that for all followers $y$ and all $j$ with guess $\rho \leqslant{ }_{L} \sigma$ and $\rho \not \subset \sigma$ (so that $\rho$ is to the left of the true path) we have that $y \in C_{j}$ iff $y \in C_{j, s_{0}}$. Since there are only finitely many such $\rho$-stages it follows that $\lim _{s} r(\rho, s)=r(\rho)$ exists. By assumption $\lim _{s} r(\tau, s)=r(\tau)$ exists for all $\tau \subset \sigma$ with $\tau \neq \sigma$. Thus it suffices to argue that $\lim _{s} r(\sigma, s)=r(\sigma)$ exists.

Now, by construction, $r(\sigma, s)$ is only reset when some follower with guess $\sigma$ is enumerated into $C_{j, s+1}-C_{j, s}$ for the sake of $R_{j, i}$ where $\langle j, i\rangle=\operatorname{lh}(\sigma)-1$. The only possible way for $\lim _{s} r(\sigma, s)$ not to exist is if $r(\sigma, s) \rightarrow \infty$ and $R_{j, i}$ receives attention infinitely often via (3.13). Thus take some follower $z$ appointed to $R_{j, i}$ after stage $s_{0}$ such that (3.13) pertains to $z$.

Let $t$ be the stage at which (3.13) pertains to $z$. We know that at some stage $t_{1}$ with $t_{1}<t$ we have that (3.15) pertained to $z$ and so $z$ was activated. At stage $t_{1}$ we know that

$$
l(\sigma, s)>M, \quad \text { where } M=\max \left\{p: p \in \operatorname{dom} \alpha_{\gamma_{i}(z)}\right\}
$$

Now since $\sigma \subset \beta$ and $s_{0}$ is chosen as above, we know that the $\hat{\Phi}_{j, t_{1}}\left(A_{t_{1}} ; y\right)$ computations for $y \leqslant M$ are $\sigma$-correct at stage $t_{1}$.

The critical observation we need to make is that at any stage $s>t_{1}$, if $l(j, s)>M$, then the $\hat{\Phi}_{j, s}\left(A_{s} ; y\right)$ computations for $y \leqslant M$ are also $\sigma$-correct. This is because the use function cannot change in a wtt-reduction. Therefore when (3.15) pertains to $z$ at stage $t$ we have that

(i) $l(j, t)>M$, and

(ii) the computations $\hat{\Phi}_{j, t}\left(A_{t} ; y\right)$ for $y \leqslant M$ are $\sigma$-correct.

Now we apply Step 2, Case 1 and this ensures that

(a) $C_{j, t+1}(z)=1$ iff $\neg W_{j, t} \vDash \alpha_{\gamma_{i}(z)}$, and

(b) $A_{t+1}[N]=A_{t}[N]$ where $N=\max \left\{\phi_{j}(y): y \leqslant M\right\}$.

We ensure (b) because we reset $r(\sigma, t)$ to be $t+1$ at stage $t+1$. Now by choice of $s_{0}$ and $\sigma \subset \beta$ we know that (b) ensures that, in fact

(b) $A[N]=A_{t}[N]=A_{t+1}[N]$.

This means that $\hat{\Phi}_{j, t}\left(A_{t} ; y\right)=\hat{\Phi}_{j}(A ; y)$ for all $y \leqslant M$. Therefore $W_{j, t}[M]=W_{j}[M]$. Thus $W_{j} \vDash \alpha_{\gamma_{i}(z)}$ iff $W_{j, t} \vDash \alpha_{\gamma_{i}(z)}$ iff $C_{j}(z)=0$. 
It is now clear that (3.14) is exactly the condition we need, since (3.14)(b) ensures that no new followers can be appointed to $R_{j, i}$ after stage $t$. Also all followers with guess $\sigma$ are cancelled in Step 1. Therefore $R_{j, i}$ never again receives attention, and so $r(\sigma, t+1)=t+1=r(\sigma)$. Thus we know that $\lim _{s} r(\sigma, s)=r(\sigma)$ exists. This easily implies that all $P_{e}$ are met: If $\sigma=\tau^{\wedge} 1$ there is nothing to prove. If $\sigma=\tau^{\wedge} 0$, then if $t$ is chosen such that $t>s_{0}$ and $r(\sigma, t)=r(\sigma)$ we see that at every $\sigma$-stage $t_{1} \geqslant t$ we have

(i) $r\left(\rho, t_{1}\right)=r(\rho, t)$ for all $\rho \leqslant{ }_{L} \sigma$ and

(ii) $r\left(\rho, t_{1}\right)=0$ for all $\rho \nless{ }_{L} \sigma_{s}$.

Thus in Step 3 any number $x$ in $D_{t}^{(e)}$, with $x>\max \left\{r(\rho): \rho \leqslant_{L} \sigma\right\}$ is enumerated into $A_{t_{1}+1}$ if $x \notin A_{t_{1}}$. Thus $D^{(e)}=* A^{(e)}$.

Finally we need argue that all the $R_{j, i}$ are met. As above let $\sigma \subset \beta$ with $\operatorname{lh}(\sigma)=\langle j, i\rangle+1$. Our earlier analysis reveals that if (3.13) ever pertains to $R_{j, i}$ after stage $s_{0}$, then $R_{j, i}$ will be met. Now if we assume that $R_{j, i}$ fails to be met, or receives attention infinitely often, it must be that $\hat{\Phi}_{j}(A)=W_{j}$ and $C_{j} \leqslant{ }_{\mathrm{tt}} W_{j}$ via $\gamma_{i}$. It is not difficult to see that $W_{j}$ is recursive since (3.13) cannot pertain after stage $s_{0}$ to any follower with guess $\sigma$ : To determine $W_{j}[z]$ find the least $\sigma$-stage $t=t(z)$ where $R_{j, i}$ has an active follower $q>z$. Since (3.13) cannot pertain to any number $\leqslant q$, we must have $W_{j}[q]=W_{j, t}[q]$. Finally if $\hat{\Phi}_{j}(A)=W_{j}$ then $C_{j} \leqslant{ }_{\text {wtt }} W_{j}$. To compute if $z \in C_{j}$ find the least $j$-expansionary stage (i.e. where $l(j, s)>l(j, t)$ for all $t<s$ ) with $W_{j, s}[z]=W_{j}[z]$. Then $z \in C_{j}$ iff $z \in C_{j, s+1}$.

4. Local structure. In this section we analyze the structure of 1- and other $r$-degrees within a single r.e. T-degree. Thus we focus on the more "local" behavior of (for example) 1-topped r.e. degrees. Various questions seem to suggest themselves. For example, we know that an r.e. degree $\mathbf{a} \neq \mathbf{0}, \mathbf{0}^{\prime}$ can have a 1-top. It would seem natural to ask if an r.e. degree $\mathbf{a} \neq \mathbf{0}$ can have a 1-bottom. This question has the following negative solution.

(4.1) THEOREM (KOBZEV [Ko1]). Each nonzero r.e. degree contains an infinite antichain of minimal r.e. btt-degrees. Here "minimal" refers to both the r.e. bttdegrees and all $\Delta_{2}^{0}$ btt-degrees.

Since a contiguous r.e. T-degree contains a single r.e. wtt-degree, some nonzero r.e. T-degrees have wtt-bottoms. The best result along these lines was also established by Kobzev [Ko3]. He constructed a strongly tt-bottomed r.e. degree $\mathbf{a} \neq \mathbf{0}$. That is, he constructed an r.e. degree $\mathbf{a} \neq \mathbf{0}$ containing an r.e. set $A$ of least tt-degree amongst all (i.e. not necessarily r.e.) sets of degree a. In fact he obtained the following result.

(4.2) TheOREM (KobzeV [Ko3]). If $A$ is an r.e. $\eta$-maximal semirecursive set, then for all $B \equiv_{\mathrm{T}} A, A \leqslant_{\mathrm{tt}} B$, and furthermore $A$ has minimal tt-degree amongst all tt-degrees.

The principal subgoal of this section is to analyze strongly tt-bottomed degrees, how they relate to 1-topped degrees and more generally, the structure of $\mathrm{tt}$-degrees within an r.e. T-degree. For our purposes (4.2) is too indirect and limiting since it 
necessarily constructs minimal tt-degrees. We first give a direct construction of a strongly tt-bottomed nonzero r.e. degree. We prove the following corollary to Kobzev's result.

(4.3) CoRollaRy (KoBzev). If $C$ is r.e. and nonrecursive, then there exist an r.e. set $A$ with $\varnothing<{ }_{\mathrm{T}} A \leqslant_{\mathrm{wtt}} C$ such that if $B$ is any set with $B \equiv_{\mathrm{T}} A$, then $A \leqslant_{\mathrm{tt}} B$.

Proof. Our construction is along the lines of Downey's [Do2] construction of a nonzero r.e. strongly contiguous degree. (Recall that $\mathbf{a}$ is strongly contiguous if $\mathbf{a}$ consists of a single wtt-degree and $\mathbf{a} \neq \mathbf{0}$.) We build $A=\mathrm{U}_{s} A_{s}$ in stages to satisfy

$$
\begin{aligned}
& P_{e}: \bar{A} \neq W_{e} . \\
& N_{e}: \text { If } \Gamma_{e}\left(\Phi_{e}(A)\right)=A \text { and } \Phi_{e}(A) \text { is total } \\
& \quad \text { then } A \leqslant{ }_{\mathrm{tt}} \Phi_{e}(A) .
\end{aligned}
$$

Here we remind the reader that $\Phi_{e}(A)$ is $\{0,1\}$-valued by convention. Before giving the formal details of the construction, we shall briefly discuss the technique we shall employ to meet the $N_{e}$.

Define

$$
l(e, s)=\max \left\{x: \forall y<x\left(\Gamma_{e, s}\left(\Phi_{e, s}\left(A_{s}\right) ; y\right)=A_{s}(y)\right)\right\} .
$$

Note implicitly here we mean that for all $z<u\left(\Gamma_{e, s}\left(\Phi_{e, s}\left(A_{s}\right) ; y\right)\right), \Phi_{e, s}\left(A_{s} ; z\right) \downarrow$.

From $N_{e}$ 's point of view, the key points regarding the $P_{e}$ will be that the $P_{e}$ are finitary in nature, and will be satisfied by followers which are always appointed at stage $s$ to be larger than $s$. In particular they exceed all computations, etc. by convention.

For a single $N_{e}$ we keep in mind that our overall aim is to achieve $A \leqslant{ }_{\mathrm{tt}} \Phi_{e}(A)$. To do this, roughly speaking, for any follower $x$ of $P_{j}$ (say) we wait till the first stage where $l(e, s)>x$. At this stage we declare $x$ as e-confirmed and attempt to ensure

$$
x \in A-A_{s} \quad \text { iff } \quad \Phi_{e, s}\left(A_{s}\right)[Q] \neq \Phi_{e}(A)[Q]
$$

where $Q=\max \left\{u\left(\Gamma_{e, s}\left(\Phi_{e, s}\left(A_{s}\right) ; y\right)\right): y \leqslant x\right\}$. (Note that this is a dtt-condition if $\Phi_{e}(A)$ is r.e.) To achieve this it is clearly in our interest to stop $\Phi_{e, s}\left(A_{s}\right)[Q]$ from changing once $x$ is $e$-confirmed (unless $x$ enters $A-A_{s}$ ). The first part of our strategy towards this aim is to cancel all lower priority followers than $x$ when $x$ is $e$-confirmed. (Such followers will be $>x$.) The net effect of this will be to ensure that there are no followers $z$ with $x<z \leqslant s$, and thus if $A_{s}[x]=A[x]$ then $\Phi_{e}(A)[Q]=\Phi_{e, s}\left(A_{s}\right)[Q]$.

By definition of a use function, we know that if $A_{s}[x] \neq A[x]$ then $\Phi_{e}(A)[Q] \neq$ $\Phi_{e, s}\left(A_{s}\right)[Q]$. Thus to complete our strategy (for a single $N_{e}$ ) we must ensure that if ever $A_{s}[x] \neq A_{t}[x]$ then $A_{s}(x) \neq A_{t}(x)$. This is fine if we add $x$ to $A_{t}-A_{t-1}$ because $x$ "receives attention," but is somewhat of a problem if some $y<x$ acts. Our solution is to use "dumping." That is, if $y \in A_{t+1}-A_{t}$ then we ensure that $\forall z\left(y \leqslant z \leqslant t \rightarrow z \in A_{t+1}\right)$. (REMARK. In the terminology of [Jo1], this makes $A$ semirecursive.) In particular, if $y$ enters $A$ then $x$ enters too. 
From a global point of view we must also incorporate various nested strategies according to whether or not $l(e, s) \rightarrow \infty$. This is again achieved by a tree of strategies argument. We now give the formal details of the argument.

A stage $s$ is defined to be a $\sigma$-stage by induction on $\ln (\sigma)$.

(i) Every stage $s$ is a $\varnothing$-stage.

(ii) If $s$ is a $\tau$-stage with $\operatorname{lh}(\tau)=e$ then if $l(e, s)>\max \{l(e, t): t$ is a $\tau$-stage and $t<s\}$, we say $s$ is a $\tau^{\wedge} 0$-stage.

Otherwise, $s$ is a $\tau^{\wedge} 1$-stage.

Let $f$ be a 1-1 recursive function enumerating a given r.e. nonrecursive set $C$ so that $f(\omega)=C$. We say that $P_{e}$ requires attention at stage $s+1$ if $W_{e, s} \cap A_{s}=\varnothing$ and one of the following options holds.

(4.4) $P_{e}$ has no follower $x$ with $x \notin W_{e, s}$.

(4.5) $P_{e}$ has a follower $x$ such that

(i) $x \in W_{e, s}$, and

(ii) $f(s) \leqslant x$.

Construction, Stage $s+1$.

Step 1. As usual, let $\sigma_{s}$ denote the unique string with $\operatorname{lh}\left(\sigma_{s}\right)=s$ and $s$ a $\sigma_{s}$-stage. Cancel all followers $x$ with guess $\tau{ }_{L} \sigma_{s}$.

Step 2. Find the least follower $x$ (if any) such that for some $\tau^{\wedge} 0 \subset \sigma_{s}$ we have

(i) $x$ has guess $\gamma$ and $\tau^{\wedge} 0 \subset \gamma$,

(ii) if $e=\operatorname{lh}(\tau)$ then $l(e, s)>x$, and

(iii) $x$ is not yet $\tau^{\wedge} 0$-confirmed.

Declare $x$ as $\tau^{\wedge} 0$-confirmed for each such $\tau$ and cancel all followers $y>x$.

REMARK. The reader should note that $\gamma \leqslant{ }_{L} \sigma_{s}$ (as $x$ is still alive) but it is not necessary for $\gamma \subset \sigma_{s}$ for Step 2 to apply.

Step 3. Find the least $e$ such that $P_{e}$ requires attention. If (4.4) holds, appoint $y=s+1$ as a follower of $P_{e}$ with guess $\sigma \subset \sigma_{s}$ where $\operatorname{lh}(\sigma)=e+1$. Cancel all followers $y$ with a guess $\gamma$ for $\gamma \supset \sigma$ and $\gamma \neq \sigma$.

If (4.5) holds, set $A_{s+1}=A_{s} \cup\{z: x \leqslant z \leqslant s\}$. $P_{e}$ is now met (forever).

END OF CONSTRUCTION.

(4.6) Lemma. (i) If $z=\mu y\left(y \in A_{s+1}-A_{s}\right)$ then $z$ is a follower.

(ii) $A \leqslant{ }_{\text {wtt }} C$.

Proof. (i) Numbers enter $A_{s+1}-A_{s}$ only in Step 3, and when (4.5) pertains to some follower $x$. We then set $A_{s+1}=A_{s} \cup\{z: x \leqslant z \leqslant s\}$.

(ii) Let $z$ be given. To decide if $z \in A$, compute the least stage $s$ such that $\forall t>s(f(t)>z)$. Then by (i) above and (ii) of (4.5) we see that $z \in A$ iff $z \in A_{s+1}$.

Now let $\beta$ denote the leftmost path. Let $\sigma \subset \beta$ with $\ln (\sigma)=e+1$.

(4.7) LeMma. $P_{e}$ receives attention finitely often at $\sigma$-stages and $P_{e}$ is met.

Proof. By induction, let $s_{0}$ be a $\sigma$-stage such that for all $s>s_{0}$

(i) For all $j<e, P_{j}$ does not receive attention at stage $s$ if $s$ is a $\sigma$-stage.

(ii) $\sigma \leqslant{ }_{L} \sigma_{s}$. 
(iii) For all $j<e, P_{j}$ does not receive attention via (4.5) at stage $s$.

(iv) No follower of $P_{j}$ for $j<e$ with guess $\tau \leqslant{ }_{L} \sigma$ receives any $\rho$-confirmation at stage $s$ (for any $\rho$ ).

Define a follower $x$ at $P_{e}$ with guess $\sigma$ to be confirmed if it is $\tau^{\wedge} 0$-confirmed for all $\tau^{\wedge} 0 \subset \sigma$. It is clear that choice of $s_{0}$ and induction ensures that if $x$ is a confirmed follower of $P_{e}$ with guess $\sigma$ appointed after stage $s_{0}$, then $x$ is uncancellable. Furthermore, (4.6)(i) ensures that $x \notin A$, unless $P_{e}$ receives attention via some follower $y \leqslant x$.

Now if $P_{e}$ fails to be met or receives attention infinitely often, it is quite easy to see that there exists an infinite recursive set of (uncancellable) followers $\left\{x_{1}<x_{2}<\right.$ $\cdots\}$ all appointed after stage $s_{0}$ such that for all $i$,

(i) $x_{i}$ is confirmed,

(ii) $x_{i}$ has guess $\sigma$, and

(iii) $x_{i} \in W_{e}$.

We claim that $C=f(\omega)$ is recursive. To compute $f(z)$ find the least stage $s>s_{0}$ such that $P_{e}$ has a confirmed follower $x_{i}>z$ with guess $\sigma$ and with $x_{i} \in W_{e, s}$. Then $z \in f(\omega)=C$ iff $z \in\{f(0), \ldots, f(s)\}$. Otherwise (4.5) would pertain to $x_{i}$ at some stage meeting $P_{e}$.

To complete the proof, we argue that $N_{e}$ is also met. Thus suppose ( $\sigma=\tau^{\wedge} 0$ with $\sigma \subset \beta$ and $\operatorname{lh}(\sigma)=e+1$. Let $s_{0}$ be a $\sigma$-stage as in the proof of (4.7). We need to show that $A \leqslant{ }_{\mathrm{tt}} \Phi_{e}(A)$ (assuming $\Phi_{e}(A)$ total). Let $z$ be given. Find the least $\sigma$-stage $s_{1}$ with $l\left(e, s_{1}\right)>z$. Suppose there is no follower $x \leqslant z$ such that $x$ has guess $\gamma \supset \sigma$. Then by (4.6)(i), choice of $s_{0}$, and cancellation at $\sigma$-stages it follows that $z \in A$ iff $z \in A_{s_{1}}$.

Thus we suppose there is some ( $\sigma$-confirmed) follower $x \leqslant z$. Let $x_{1}$ be the largest such follower. Let

$$
Q=\max \left\{u\left(\Gamma_{e, s_{1}}\left(\Phi_{e, s_{1}}\left(A_{s_{1}}\right) ; y\right)\right): y \leqslant x_{1}\right\} .
$$

We claim that if $z \notin A_{s_{1}}$, then

$$
z \in A \quad \text { iff } \quad \Phi_{e}(A)[Q] \neq \Phi_{e, s_{1}}\left(A_{s_{1}}\right)[Q] .
$$

Note that (4.8) is a tt-condition. (Also if $\Phi_{e}(A)$ is r.e. then this is a dtt-condition. In any case it is a dtt-condition from $\Phi_{e}(A) \oplus \overline{\Phi_{e}(A)}$.

Certainly as $x_{1}$ is the largest $\sigma$-confirmed follower $\leqslant z$ at stage $s$, the way we appoint followers ensures that

$$
\text { if } A_{s_{1}}\left[x_{1}\right]=A\left[x_{1}\right] \text { then } A_{s_{1}}\left[s_{1}\right]=A\left[s_{1}\right] \text {. }
$$

(By (4.6)(i) and minimality of $s_{1}$.) Therefore if $A_{s_{1}}\left[x_{1}\right]=A\left[x_{1}\right]$ then

$$
\Phi_{e, t}\left(A_{t}\right)[Q]=\Phi_{e, s_{1}}\left(A_{s_{1}}\right)[Q] \text { for all } t \geqslant s_{1},
$$

since $l\left(e, s_{1}\right)>z$ at stage $s_{1}$. By (4.9) and (4.6)(i) and the construction, we have

$$
A_{s_{1}}(z)=A(z) \text { implies } \Phi_{e, s_{1}}\left(A_{s_{1}}\right)[Q]=\Phi_{e}(A)[Q] .
$$

Conversely, suppose $A_{s_{1}}(z) \neq A(z)$. Then by construction and (4.6)(i), $A_{s_{1}}\left[x_{1}\right] \neq$ $A\left[x_{1}\right]$. Now since $\Gamma_{e, s_{1}}\left(\Phi_{e, s_{1}}\left(A_{s_{1}}\right) ; x_{1}\right)=0 \neq A\left(x_{1}\right)$ it cannot be that $\Phi_{e}(A)[Q]=$ $\Phi_{e, s_{1}}\left(A_{s_{1}}\right)[Q]$ by definition of $Q$. Thus $\Phi_{e}(A)[Q] \neq \Phi_{e, s_{1}}\left(A_{s_{1}}\right)[Q]$. Putting this together with (4.10) gives (4.8), as required, and so $A \leqslant{ }_{\mathrm{tt}} \Phi_{e}(A)$. 
As we remarked at various points throughout the argument this construction gives (apparently) a little more than Kobzev's result:

(4.11) Corollary. Let $C$ be r.e. and nonrecursive. Then there exists an r.e. set $A$ with $\varnothing<{ }_{\mathrm{T}} A \leqslant{ }_{\text {wtt }} C$ such that

(i) $B \equiv{ }_{\mathrm{T}} A$ and $B$ r.e. implies $A \leqslant{ }_{\mathrm{dtt}} B$, and

(ii) furthermore for any set $D \equiv{ }_{\mathrm{T}} A, A \leqslant{ }_{\mathrm{dtt}} D \oplus \bar{D}$.

We remark that all known constructions of (strongly) tt-bottomed r.e. degrees seem to use semirecursive sets such as $A$ above. We do not know if this is necessary. Concerning the T-degrees of tt-bottomed r.e. degrees, we do not know of any jump class classification although it seems probable that no high r.e. degree can be tt-bottomed. Later we show $\mathbf{0}^{\prime}$ is not wtt-bottomed. In particular, the index set reasoning we used for 1-degrees in $\S 3$ fails since, as we show, $\left\{e: W_{e} \geqslant{ }_{\text {wtt }} A\right\}$ is always different from $\left\{e: W_{e} \geqslant{ }_{\mathrm{T}} A\right\}$ for $A$ r.e., nonrecursive, and incomplete.

We do have a partial classification in terms of wtt-cuppability. Recall that an r.e. set $A$ is wtt-cuppable if there exists an r.e. set $B$ such that $A \oplus B \equiv{ }_{\text {wtt }} \varnothing^{\prime}$ and $\varnothing^{\prime} \nless{ }_{\mathrm{wtt}} B$.

E. L. Post showed [Po] that hypersimple sets cannot be tt-complete, and R. Friedberg and H. Rogers observed [FR, p. 124] that similar methods may be used to show that hypersimple sets cannot be wtt-complete. In the next theorem we extend this to show that no hypersimple set can be wtt-cuppable. This result will have as a corollary a result mentioned in [AJSS, p. 124] : no contiguous degree is wtt-cuppable. We thank Jeanleah Mohrherr for asking about the connection between hypersimplicity and wtt-cuppability.

\section{(4.12) THEOREM. No hypersimple set is wtt-cuppable.}

Proof. Let $H$ be hypersimple and $A$ be r.e. Assume that $K \leqslant{ }_{\text {wt }} H \oplus A$, where $K$ is creative. We must show that $K \leqslant{ }_{\text {wtt }} A$. Using the technique devised by Lachlan for the nondiamond theorem [La1, Theorem 5], we enumerate an r.e. set $E$ in such a way as to "force" many numbers into $H \oplus A$, and ultimately into $A$. Since $E \leqslant{ }_{\text {wtt }} H \oplus A$ and our construction is uniform, we may assume that we know in advance a wtt-reduction procedure $\hat{\Gamma}$ such that $E=\hat{\Gamma}(H \oplus A)$, together with its (recursive) use function $\gamma$. Let

$$
l(s)=\max \left\{x:(\forall y<x)\left[\hat{\Gamma}_{s}\left(H_{s} \oplus A_{s} ; y\right)=E_{s}(y)\right]\right\} .
$$

Clearly if $u<l(s), u<l(t)$, and $u \in E_{t}-E_{s}$, then

$$
\left(H_{t} \oplus A_{t}\right)[\gamma(u)] \neq\left(H_{s} \oplus A_{s}\right)[\gamma(u)] .
$$

We may assume without loss of generality that $\gamma$ is nondecreasing and that $\langle n, j\rangle$ is nondecreasing as a function of $j$, so that $\gamma(\langle n, j\rangle)$ is a nondecreasing function of $j$.

If $n$ enters $K$, we subsequently put the numbers $\langle n, 0\rangle,\langle n, 1\rangle,\langle n, 2\rangle, \ldots$ into $E$ in that order, but we do not put $\langle n, j+1\rangle$ into $E$ until the change in $H \oplus A$ below $\gamma(\langle n, j\rangle)$-guaranteed by (4.13) with $u=\langle n, j\rangle$-has already occurred. More 
precisely, we define $E=\bigcup_{s} E_{s}$, where $E_{0}=\varnothing$ and

$$
\begin{aligned}
E_{s+1}=E_{s} \cup\left\{\langle n, k\rangle: n \in K_{s} \&\langle n, k\rangle<l(s)\right. \\
\left.\quad \&(\forall j<k)\left[\langle n, j\rangle \in E_{s}\right]\right\} .
\end{aligned}
$$

It is trivial to show by induction on $k$ that if $n \in K$, then $\langle n, k\rangle \in E$ for all $k$ (so that $E=K \times \omega)$. For fixed $n \in K$, let $t(k)$ be the unique $s$ with $\langle n, k\rangle \in E_{s+1}-$ $E_{s}$. By (4.13)

$$
\left(H_{t(k)} \oplus A_{t(k)}\right)[(\gamma\langle n, k\rangle)] \neq\left(H_{t(k+1)} \oplus A_{t(k+1)}\right)[\gamma(\langle n, k\rangle)] .
$$

Using the above, the assumption that $\gamma(\langle n, k\rangle)$ is nondecreasing in $k$, and the r.e.-ness of $H$ and $A$, it follows that $\left(H_{s} \oplus A_{s}\right)[\gamma(\langle n, k\rangle)]$ has at least $k+1$ distinct values for $s \in[t(0), t(k+1)]$. Suppose for the moment that $\bar{H}_{s}$ has at most $k$ elements $\leqslant \gamma(\langle n, k\rangle)$. Then

$$
A_{t(0)}[\gamma(\langle n, k\rangle)] \neq A[\gamma(\langle n, k\rangle)]
$$

since not all of the changes in $H \oplus A$ can be due to changes in $H$.

The assumption that $H$ is hypersimple is used to show that the hypothesis of (4.14) is satisfied sufficiently often. Let any number $n$ be given. (We no longer assume that $n \in K$ !) Then there exist $k$ and $s$ such that $\bar{H}_{s}$ has at most $k$ elements $\leqslant \gamma(n, k)$. (Otherwise $\bar{H}$ has $>k$ elements $\leqslant \gamma(\langle n, k\rangle)$ for all $k$, in contradiction to the domination definition of hypersimplicity. We remind the reader that this definition reads that $A$ is hypersimple if its complement is not majorized by any recursive function (see [Rg, p. 139]).) Let $\left\langle k_{n}, s_{n}\right\rangle$ be (say) the least pair $\langle k, s\rangle$ with this property. To compute whether $n \in K$ from $A$, find $\hat{s}$ so large that

$$
A_{\hat{s}}\left[\gamma\left(\left\langle n, k_{n}\right\rangle\right)\right]=A\left[\gamma\left(\left\langle n, k_{n}\right\rangle\right)\right] .
$$

Then $n \in A$ iff $n \in A_{\hat{s}}$. To verify this, assume for a contradiction that $n \in A-A_{\hat{s}}$. Then $t(0)\rangle \hat{s}$ where, as before, $t(k)=(\mu s)\left[\langle n, k\rangle \in E_{s+1}\right]$. By (4.14) with $k=k_{n}$, $A_{t(0)}\left[\gamma\left(\left\langle n, k_{n}\right\rangle\right)\right] \neq A\left[\gamma\left(\left\langle n, k_{n}\right\rangle\right)\right]$, so $A_{\hat{s}}\left[\gamma\left(\left\langle n, k_{n}\right\rangle\right)\right] \neq A\left[\gamma\left(\left\langle n, k_{n}\right\rangle\right)\right]$. This contradicts the choice of $\hat{s}$. Thus $K \leqslant{ }_{\text {wtt }} A$ with use function $\gamma\left(\left\langle n, k_{n}\right\rangle\right)$.

(4.15) COROLlary. Every r.e. T-degree contains an r.e. set which is not wtt-cuppable. In particular, no contiguous r.e. degree a contains a wtt-cuppable set.

REMARK. The latter result was mentioned without proof in [AJSS, p. 124], and a direct proof is given in [AS, Theorem 5.3].

Proof. Let $\mathbf{a}$ be any r.e. degree. If $\mathbf{a}=\mathbf{0}$, then the result is trivial. If $\mathbf{a} \neq \mathbf{0}$, then $\mathbf{a}$ contains a hypersimple set by [De]. The second sentence of the corollary follows immediately from the first since wtt-cuppability is invariant under $\equiv{ }_{\text {wtt }}$.

Actually, the proof that contiguous degrees are not wtt-cuppable which is given in [AS] establishes somewhat more. This proof shows that wtt-bottomed r.e. degrees contain r.e. sets that are not wtt-cuppable. In particular, the wtt-bottoms are not wtt-cuppable. This result also follows from (4.12) in the manner of (4.15) since we know that if $A$ is any r.e. nonrecursive set then there exists a hypersimple r.e. set $B \equiv{ }_{\mathrm{T}} A$ with $B \leqslant{ }_{\text {wtt }} A$. The easiest way to see this is to consider the Dekker [De] deficiency set for $B$. (This actually achieves $B \leqslant_{\mathfrak{t}} A$, see (e.g.) Soare [So4, V, Ex. 2.12].) 
We now turn to the relationships between $r$-topped degrees and tt-bottomed ones. The fact that nonzero strongly contiguous r.e. degrees exist would seem to suggest that perhaps there exists an r.e. degree consisting of a single (r.e.) tt-degree. This suggestion fails by Cohen [Co]. In fact, building on earlier results of Lachlan [La2] and Cohen [Co], Kobzev [Ko2] has shown that each nonzero r.e. wtt-degree contains an infinite antichain of r.e. $\mathrm{tt}$-degrees. By our results of $\S 3$ we have the following.

(4.16) THEOREM. Below any nonzero r.e. degree there exists a nonzero r.e. strongly tt-bottomed T-degree which is not tt-topped.

Proof. Combine (4.3) with (3.3).

We can do a little better by squeezing more information out of the construction of (4.3).

(4.17) THEOREM. The r.e. set A constructed in (4.3) is also of strongly contiguous degree. Thus, if $C$ is any r.e. nonrecursive set there exists an r.e. set $A$ with $\varnothing<{ }_{\mathrm{T}} A \leqslant{ }_{\mathrm{wtt}} C$ such that for all sets $B$, if $B \equiv{ }_{\mathrm{T}} A$ then $A \leqslant{ }_{\mathrm{tt}} B$ and $B \leqslant{ }_{\mathrm{wtt}} A$. Hence $\operatorname{deg}(A)$ is strongly contiguous and tt-bottomed.

Proof. We verify that (4.3) gives the desired result. Thus suppose $\Gamma_{e}\left(\Phi_{e}(A)\right)=A$. We also claim that $\Phi_{e}(A) \leqslant{ }_{\text {wtt }} A$.

Let $\sigma, s_{0}$ be as in (4.7) and (4.8). (Recall $\sigma \subset \beta$ with $\operatorname{lh}(\sigma)=e+1$ and $s_{0}$ is a stage "good for $\sigma$ ".) Let $x$ be given. We show how to compute $\Phi_{e}(A)(x)$ from $A$. Find the least $\sigma$-stage that $l\left(e, s_{1}\right)>x$ and $s_{1}>s_{0}$. Now compute the least $\sigma$-stage $s_{2}>s_{1}$ with

$$
A_{s_{2}}\left[s_{1}\right]=A\left[s_{1}\right] \text {. }
$$

We claim that $\Phi_{e, s_{2}}\left(A_{s_{2}}\right)(x)=\Phi_{e}(A)(x)$. We give the details although the argument is fairly standard by now. Suppose not. By (4.18), this means that there is some number $y$ with

$$
s_{1} \leqslant y<u\left(\Phi_{e, s_{2}}\left(A_{s_{2}} ; x\right)\right)
$$

such that $y$ enters $A$ after stage $s_{2}$. By (4.6)(i) and (4.18) we may clearly suppose $y$ is a follower at the stage $t$ when $y \in A_{t}-A_{s_{2}}$. By cancellation at $\sigma$-stages, $y$ must have guess $\gamma \supset \sigma$ since it is still alive at stage $s_{2}$. Now since $y$ has guess $\supset \sigma$ it must have been appointed at a $\sigma$-stage $s_{3}$ with $s_{1} \leqslant s_{3}<s_{2}$. Since $y \notin A_{s_{2}}$ no number $\leqslant y$ has entered $A$ after stage $s_{3}$ but before stage $s_{2}$. (The entry of such a number would cause $y$ to also enter.) It follows that $A_{s_{3}}\left[s_{1}\right]=A_{s_{2}}\left[s_{1}\right]$ since $s_{1} \leqslant y$. But then $A_{s_{3}}\left[s_{1}\right]=A\left[s_{1}\right]$ by (4.18). This specifically contradicts the minimality of $s_{2}$ since $s_{3}<s_{2}$. Hence $A\left[u\left(\Phi_{e, s_{2}}\left(A_{s_{2}} ; x\right)\right)\right]=A_{s_{2}}\left[u\left(\Phi_{e, s_{2}}\left(A_{s_{2}} ; x\right)\right)\right]$ and so $\Phi_{e, s_{2}}\left(A_{s_{2}}\right)(x)=$ $\Phi_{e}(A)(x)$ as desired. Therefore $\Phi_{e}(A) \leqslant{ }_{\mathrm{wtt}} A$.

(4.18) COROLlary. There exist r.e. T-degrees consisting of a single wtt-degree that are strongly tt-bottomed, but have no tt-top.

On the other hand, in $\S 3$ we observed that an r.e. degree may be 1-topped but not tt-bottomed. For $\mathbf{0}_{\mathrm{T}}^{\prime}$ in fact we have

(4.19) THEOREM. $\mathbf{0}_{\mathrm{T}}^{\prime}$ is 1-topped but if $R$ is any r.e. set wt below all r.e. sets of $T$-degree $\mathbf{0}^{\prime}$ then $R$ is recursive. 
Proof. Suppose $\varnothing<{ }_{\mathrm{T}} R$ and $R$ satisfies the given hypotheses. We build a T-complete r.e. set $A$ with $R{ }_{\text {wtt }} A$. Thus let $f(\omega)$ be a 1-1 enumeration of a creative r.e. set. We satisfy the requirements $Q_{e}: \hat{\Gamma}_{e}(A) \neq R$. Here $\hat{\Gamma}_{e}$ denotes the $e$ th wtt-reduction and has monotone use $\gamma_{e}$. Now let

$$
\begin{aligned}
& l(e, s)=\max \left\{x: \forall y<x\left(\hat{\Gamma}_{e, s}\left(A_{s} ; y\right)=R_{s}(y)\right)\right\}, \\
& m l(e, s)=\max \{l(e, s): t<s\}, \text { and } \\
& l s(e, s)=\max \{0, t: t \text { is a stage }<s \text { with } l(e, t)>\operatorname{ml}(e, t)\} .
\end{aligned}
$$

We say that $Q_{e}$ requires attention at stage $s+1$ if $e$ is least such that $l(e, s)>$ $m l(e, s)$.

CONSTRUCTION.

StaGe 0. Set $A_{0}=\varnothing$ and $a_{i, 0}=i$ for all $i \in \omega$.

STAGE $s+1$. Find the $e$ (if any) such that $Q_{e}$ requires attention. Let

$$
n= \begin{cases}\min \{f(s), e\} & \text { if } e \text { exists, } \\ f(s) & \text { otherwise. }\end{cases}
$$

Set

$$
A_{s+1}=A_{s} \cup\left\{a_{n, s}, \ldots, a_{n+s, s}\right\},
$$

and set

$$
a_{e, s+1}= \begin{cases}a_{i, s} & \text { for } i<n, \\ a_{i+n+s+1, s} & \text { otherwise. }\end{cases}
$$

END OF CONSTRUCTION.

VERIFICATION. We argue that $\lim _{s} a_{i, s}=a_{i}$ exists and each $Q_{e}$ receives attention at most finitely often (and is met). For an induction, let $s_{0}$ be a stage such that for all $s>s_{0}$

(i) $\forall j<e\left(Q_{j}\right.$ does not receive attention at stage $\left.s\right)$,

(ii) $f(s)>e$, and

(iii) $a_{j, s}=a_{j}$ for $j<e$.

Suppose that $Q_{e}$ receives attention infinitely often. Then $l(e, s) \rightarrow \infty$. We show that this implies that $R$ is recursive.

To compute $R(x)$ find the least stage $s_{1}>s_{0}$ with $l\left(e, s_{1}\right)>m l\left(e, s_{1}\right)$ and $l\left(e, s_{1}\right)>x$. Then $Q_{e}$ receives attention at stage $s_{1}$ and

$$
A_{s+1}=A_{s_{1}} \cup\left\{a_{e, s_{1}}, \ldots, a_{e+s_{1}, s_{1}}\right\} .
$$

Now by convention $\gamma_{e}(x)<s_{1}$. By induction $a_{i, s_{1}} \geqslant i$. Thus we see

$$
\forall z\left(\left(z<\gamma_{e}(x) \& z \notin A_{s_{1}+1}\right) \rightarrow z<a_{e, s_{1}}\right) .
$$

The assumptions on $s_{0}$ imply that this means

$$
\forall z\left(\left(z<\gamma_{e}(x) \& z \in A\right) \rightarrow z \in A_{s_{1}}\right) .
$$

Consequently, if $s_{2}$ is the least stage with $s_{2}>s_{1}$ and $l\left(e, s_{2}\right)>m l\left(e, s_{2}\right)$ we see that the computation $\Phi_{e, s_{2}}\left(A_{s_{2}} ; x\right)$ is final. Hence $x \in R$ if $x \in R_{s_{2}}$. Hence $R$ is recursive. Therefore $Q_{e}$ receives attention finitely often. This in turn means $\lim _{s} a_{e, s}$ $=a_{e}$ exists since after stage $s_{0}, a_{e, s}$ can only change when $Q_{e}$ receives attention. 
Finally, we need argue that $A \equiv{ }_{\mathrm{T}} f(\omega)$. To compute if $x \in f(\omega) A$-recursively find the least stage $s$ such that $a_{x, s}=a_{x, t}$ for all $t>s$. Then $x \in f(\omega)$ iff $x \in\{f(0), \ldots, f(s)\}$.

For our next results we establish that an r.e. degree can be strongly tt-bottomed but have no wtt-top. To complete the picture we would need to construct an r.e. degree $\mathbf{a} \neq \mathbf{0}$ with a 1-top and a (strong) tt-bottom. We do not know how to do this! In fact, it is an open question whether there exists an r.e. degree $\mathbf{a} \neq \mathbf{0}$ with an r.e. tt-top and an r.e. wtt-bottom. The infinitary nature of the positive requirements used to satisfy the 1-top requirements of (2.1) seem to interfere very strongly with the delicate contiguity-type machinery used to construct wtt-bottoms. On the other hand this machinery can be modified to admit certain infinitary positive requirements as witnessed by Ladner's [Ld] difficult construction of a $\operatorname{low}_{2}-\operatorname{low}_{1}$ "completely mitotic" contiguous r.e. degree.

(4.20) THEOREM. There exists an r.e. degree a such that a is strongly tt-bottomed but has no wtt-top. In fact for all (not necessarily r.e.) sets $B$ of degree a there is an r.e. set $C$ of degree a with $C{ }_{\mathrm{wtt}} B$.

Proof. We build $A=\bigcup_{s} A_{s}$ together with auxiliary r.e. sets $C_{e}=\bigcup_{s} C_{e, s}$ to satisfy

$$
\begin{aligned}
& R_{e, i}: \Gamma_{e}\left(\Phi_{e}(A)\right)=A \text { implies } C_{e} \leqslant{ }_{\mathrm{T}} A \text { and } \hat{\Phi}_{i}\left(\Phi_{e}(A)\right) \neq C_{e} . \\
& N_{e}: \Gamma_{e}\left(\Phi_{e}(A)\right)=A \text { implies } A \leqslant{ }_{\mathrm{tt}} \Phi_{e}(A) .
\end{aligned}
$$

Again, let

$$
l(e, s)=\max \left\{x: \forall y<x\left(\Gamma_{e, s}\left(\Phi_{e, s}\left(A_{s}\right) ; y\right)=A_{s}(y)\right)\right\} .
$$

Now as in (4.3) define " $\sigma$-stage" by induction on $\operatorname{lh}(\sigma)$ using $l(e, s)$ : every stage is a $\varnothing$-stage and if $s$ is a $\tau$-stage with $\operatorname{lh}(\tau)=e$ define $s$ to be a $\tau^{\wedge} i$ stage where $i=0$ if $l(e, s)>\max \{l(e, t): t<s\}$ and $i=1$ otherwise.

Define $\sigma_{s}$ as usual. Also define $m l(e, s)=\max \{l(e, t): t<s\}$. We say a stage $s$ is $e$-expansionary if $l(e, s)>m l(e, s)$. Now, define

$$
\begin{array}{r}
L(e, i, s)=\max \left\{x: \forall y<x\left(\hat{\Phi}_{i, s}\left(\Phi_{e, s}\left(A_{s}\right) ; y\right)=C_{e, s}(y)\right.\right. \\
\left.\left.\& l(e, s)>\phi_{i, s}\left(\Phi_{e, s}\left(A_{s}\right) ; y\right)\right)\right\} .
\end{array}
$$

Here the reader should recall that $\phi_{i}$ is the use of $\hat{\phi}_{i}$ by convention (and is nondecreasing). We say a stage $s$ is (e,i)-expansionary if $L(e, i, s)>m L(e, i, s)$ where $m L(e, i, s)=\max \{L(e, i, t): t<s\}$.

We briefly describe the method we employ to satisfy the $R_{e, i}$ in conjunction with the $N_{e}$. First for the $N_{e}$ we proceed almost exactly as we did in (4.3). Of course we cannot proceed exactly as we did since we know that this gives a strongly contiguous degree. The crucial difference will be that we will allow numbers to be appointed to some $R_{e, i}$ at some guess $\sigma$ with $\operatorname{lh}(\sigma)=\langle e, i\rangle$ at other than $\sigma$-stages. (These numbers will be traces and $R_{e, i}$ will be "waiting".)

We satisfy the $R_{e, i}$ by a Friedberg-Muchnik type procedure. Basically we wait till $L(e, i, s)>x$ for some follower $x$ of $R_{e, i}$ targeted for $C_{e}$. At this stage we enumerate $x$ into $A$ and declare $R_{e, i}$ as waiting. "Waiting" indicates that whenever possible we 
wish to give $x$ a trace (to ensure $C_{e} \leqslant{ }_{\mathrm{T}} A$ ). The problem is that we cannot immediately give $x$ a trace $T(x)$ targeted for $A$. Our idea will be to be able to add $x$ to $C_{e}$ and $T(x)$ to $A$ at the same time to create a disagreement. Thus, we must know that $T(x)$ 's entry will be good from the point of view of not injuring " $\hat{\Phi}_{e, s}\left(\Phi_{e, s}\left(A_{s}\right) ; x\right)=0$ " computations. Now when $x$ entered $A_{t}$ the " $\Phi_{e, t}\left(A_{t} ; x\right)$ " computations may have been injured and perhaps the use changed. Therefore we must wait until the " $\Phi_{e, t}\left(A_{t} ; x\right)$ " computations recover before we know how big the use is, and so how large $T(x)$ needs to be. Thus, we choose to wait until the next $e$-expansion stage $s>t$ and set $T(x)=s+1$. We note that this is fine because $C_{e} \leqslant{ }_{\mathrm{T}} A$ is predicated upon $\Gamma_{e}\left(\Phi_{e}(A)\right)=A$.

Now at stage $s$ when $T(x)$ is set, perhaps $s$ is not a $\sigma$-stage where $x$ has guess $\sigma$. Nevertheless, $T(x)$ will inherit $x$ 's guess (so $T(x)$ has guess $\sigma$ ) to cooperate with the $N_{j}$ requirement.

The remainder of the argument is straightforward. We declare $x$ as active and wait till $L(e, i, s)>x$ again. Then we create a disagreement by setting $C_{e, s+1}=C_{e, s} U$ $\{x\}$ and enumerating $T(x)$ into $A$.

Formal details now follow, although we suspect that the reader may wish to supply them himself.

We say that $R_{e, i}$ requires attention at stage $s+1$ if $R_{e, i}$ is not currently declared satisfied. One of the following options holds.

(4.21) $R_{e, i}$ is active via $x$ and $s$ is $(e, i)$-expansionary.

(4.22) $R_{e, i}$ is waiting via $x$ and $s$ is $e$-expansionary.

(4.23) $R_{e, i}$ is inactive but has a follower $x$ with $L(e, i, s)>x$ and $s$ is $(e, i)$-expansionary.

(4.24) $R_{e, i}$ is inactive, and has no follower.

Construction, Stage $s+1$.

Step 1. Let $\sigma_{s}$ denote the unique string with $\operatorname{lh}\left(\sigma_{s}\right)=s$ and $s$ a $\sigma_{s}$-stage. Cancel all followers or traces $x$ with guesses $\tau{ }_{L} \sigma_{s}$. Also for each $e, i$ if $R_{e, i}$ is active or waiting via such $x$, declare $R_{e, i}$ as inactive.

Step 2. Find the least follower or trace $z$ not already $\tau^{\wedge} 0$-confirmed for some $\tau^{\wedge} 0 \subset \sigma_{s}$ such that

(i) $z$ has guess $\gamma \supset \tau^{\wedge} 0$, and

(ii) $l(e, s)>z$, where $e=\operatorname{lh}(\tau)$.

Declare $z$ as $\tau^{\wedge} 0$-confirmed for each such $\tau^{\wedge} 0$ and cancel all followers or traces $z^{\prime}>z$.

Step 3. Find the least $\langle e, i\rangle$ (if any) such that $R_{e, i}$ requires attention. If none exists go to stage $s+2$. Let $\sigma \subset \sigma_{s}$ with $\operatorname{lh}(\sigma)=\langle e, i\rangle+1$. Cancel all followers or traces with guesses $\gamma \supset \sigma$ and $\gamma \neq \sigma$. Adopt the appropriate case below.

Case 1. (4.21) holds. Declare $R_{e, i}$ as currently satisfied via $x$. Set $A_{s+1}=A_{s} \cup$ $\{z: T(x) \leqslant z \leqslant s\}$. Set $C_{e, s+1}=C_{e, s} \cup\{x\}$. (This step temporarily satisfies $R_{e, i}$.) Cancel all followers or traces of $R_{g}$ for $g>e$ and declare these as inactive.

Case 2. (4.22) holds. Declare $R_{e, i}$ as active via $x$ and set $T(x)=s+1$. Declare $T(x)$ to have guess $\gamma$ where $\gamma$ is the guess of $x$. (Note $T(x)$ is not yet $\rho$-confirmed for any $\rho$.) Cancel all followers or traces with guess $\eta$ for $\eta{ }_{L} \gamma$. Declare the appropriate $R_{g}$ as inactive. 
Case 3. (4.23) holds. Declare $R_{e, i}$ as waiting. Set $A_{s+1}=A_{s} \cup\{z: x \leqslant z \leqslant s\}$. Cancel all followers or traces of $R_{g}$ for $g>e$. Declare these $R_{g}$ as inactive.

Case 4. (4.24) holds. Appoint $x=s+1$ as a follower of $R_{e, i}$ with guess $\sigma$. Cancel all followers and traces with guess $\gamma$ for $\gamma \supset \sigma$ and $\gamma \neq \sigma$. For any $R_{g}$ corresponding to these followers or traces, declare $R_{g}$ as inactive.

END OF CONSTRUCTION.

Verification. Let $\beta$ denote the leftmost path. Let $\sigma \subset \beta$ with $\operatorname{lh}(\sigma)=\langle e, i\rangle$. For an induction, suppose that $s_{0}$ is a $\sigma$-stage such that for all $s>s_{0}$ we have:

(i) For all $k<\langle e, i\rangle, R_{k}$ does not receive attention at stage $s$, nor do any numbers associated with $R_{k}$ receive any confirmation at stage $s$.

(ii) $\sigma \leqslant{ }_{L} \sigma_{s}$.

(iii) All followers or traces with guess $\tau \ngtr{ }_{L} \sigma$ cease acting.

We now verify that $R_{e, i}$ receives attention at most finitely often and is met. After stage $s_{0}$, once $R_{e, i}$ gets a follower $x$ with guess $\gamma \leqslant{ }_{L} \sigma$ this follower $x$ is evidently uncancellable. We claim that this follower succeeds in meeting $R_{e, i}$. Notice that such $x$ will be the last follower $R_{e, i}$ ever receives. This follows since either we get stuck in some state such as waiting, or via $x, R_{e, i}$ eventually is declared satisfied. This declaration can only be cancelled by higher priority activity, which cannot happen by choice of $s_{0}$. Thus for the $R_{e, i}$ it remains to verify that the strategy actually works.

Thus suppose $\Gamma_{e}\left(\Phi_{e}(A)\right)=A$. This clearly means that (4.23) pertains to $x$ at some stage $s_{1}$, say. At this stage, we know $L\left(e, i, s_{1}\right)>x$ and in particular

$$
\hat{\Phi}_{i, s_{1}}\left(\Phi_{e, s_{1}}\left(A_{s_{1}}\right) ; x\right)=C_{e, s_{1}}(x)=0 .
$$

Thus at stage $s_{1}+1$, we enumerate $x$ into $A_{s_{1}+1}-A_{s_{1}}$, and declare $R_{e, i}$ as waiting. Now at the first $e$-expansionary stage $s_{2}$ exceeding $s_{1}$ (4.22) pertains and we set $T(x)=s_{2}+1$. By convention this means

$$
T(x)>u \text { for } u=\max \left\{u\left(\Phi_{e, s_{2}}\left(A_{s_{2}} ; z\right)\right): z \leqslant \phi_{e}(x)\right\} .
$$

Furthermore, the cancellation procedure and choice of $s_{0}$ will ensure that

$$
\Phi_{e, s_{2}}\left(A_{s_{2}}\right)[u]=\Phi_{e}(A)[u] \quad \text { (with } u \text { as in (4.26)). }
$$

Now, either (4.21) never pertains to $x$ and so $l(e, i, s) \nrightarrow \infty$, or (4.21) pertains and (4.25), (4.26) and (4.27) together ensure that then

$$
\hat{\Phi}_{i}\left(\Phi_{e}(A) ; x\right)=0 \neq 1=C_{e}(x) .
$$

Finally, $C_{e} \leqslant{ }_{\mathrm{T}} A$ by traces. To decide if $x \in C_{e}$, see if $x$ is a follower of some $R_{e, i}$ by stage $x+1$. If $x \notin A_{x}$ and $x$ is not a follower by stage $x+1$ then $x \notin C_{e}$. If $x$ is a follower, then $x \in C_{e}$ only if $x \in A$. If $x \in A$ find the stage $s$ when this occurs. Now at the least $e$-expansionary stage $s_{1}>s$ either $x$ has been cancelled or $x$ has a trace $T(x)$ at stage $s_{1}+1$. Finally if $x$ is still alive, then $x \in C_{e}$ only if $T(x) \in A$. If $T(x) \in A$ go to stage $s_{2}>s_{1}$ where $T(x) \in A_{s_{2}+1}-A_{s_{2}}$. Then $x \in C_{e}$ iff $x \in$ $C_{e, s_{2}+1}$.

The verification for the $N_{e}$ is almost the same as in (4.3). For example (4.6)(i) now reads "if $z=\mu y\left(y \in A_{s+1}-A_{s}\right)$ then $z$ is either a follower or a trace". Thus we ask the reader to check for himself that these details go through unchanged save for minor changes like the above. 
We point out that again this argument blends with permitting to construct such an r.e. degree below any given nonzero r.e. degree. We remark that to answer our question "does there exist a tt-topped and tt-bottomed r.e. degree $\neq \mathbf{0}$ ?" does not necessitate the construction of a contiguous degree with these properties. It is possible for an r.e. degree $\mathbf{a} \neq \mathbf{0}$ to be noncontiguous and yet wtt-topped and bottomed. This is witnessed by the next result which also has several nice corollaries.

(4.28) TheOREM. (a) Let $C \not \equiv{ }_{\mathrm{T}} \varnothing$ be r.e. Then there exist r.e. sets $A$ and $B$ with $A \equiv{ }_{\mathrm{T}} B$ and $A \leqslant{ }_{\text {wtt }} C$ such that

(i) for all r.e. sets $D$ if $D \leqslant{ }_{\mathrm{T}} B$ then $D \leqslant{ }_{\mathrm{wtt}} A$,

(ii) for all sets $E$ if $B \equiv_{\mathrm{T}} E$ then $B \leqslant_{\mathrm{tt}} E$, and

(iii) $A \not \equiv{ }_{\text {wtt }} B$.

(b) Hence $\operatorname{deg}(A)$ is wtt-topped and strongly tt-bottomed but not contiguous.

Proof. For simplicity, we drop the $A \leqslant{ }_{\text {wtt }} C$ requirements which are achieved by an easy permitting argument along the lines considered earlier. Also, since the argument is essentially an amalgam of earlier ones ((4.3) and (4.17)) and a wait-andsee argument, we feel fairly free to merely sketch some details, and also not to discuss the various strategies. We build $A=\cup_{s} A_{s}$ and $B=\cup_{s} B_{s}$ to satisfy

$$
\begin{aligned}
& N: A \equiv{ }_{\mathrm{T}} B . \\
& R_{e}: \Phi_{e}(A)=W_{e} \text { implies } W_{e} \leqslant{ }_{\mathrm{wtt}} A . \\
& N_{e}: \Gamma_{e}\left(\Phi_{e}(B)\right)=B \text { implies } \Phi_{e}(B) \geqslant_{\mathrm{tt}} B . \\
& P_{e}: \hat{\Phi}_{e}(B) \neq A .
\end{aligned}
$$

We ensure $A \equiv{ }_{\mathrm{T}} B$ by traces. Numbers may be targeted for $A$ or $B$ or both. Let

$$
l(e, s)=\max \left\{x: \forall y<x\left(\Phi_{e, s}\left(A_{s} ; y\right)=W_{e, s}(y)\right)\right\},
$$

and let

$$
L(e, s)=\max \left\{x: \forall y<x\left(\Gamma_{e, s}\left(\Phi_{e, s}\left(B_{s}\right) ; y\right)=B_{s}(y)\right)\right\} .
$$

We associate $R_{e}$ with those $\sigma \in 2^{<\omega}$ with $\operatorname{lh}(\sigma)=2 e+1$. We associate $N_{e}$ with those $\sigma \in 2^{<\omega}$ with $\operatorname{lh}(\sigma)=2 e+2$. A stage $s$ is called a $\sigma$-stage by induction on $\ln (\sigma)$.

(i) Every stage $s$ is a $\varnothing$-stage.

(ii) If $s$ is a $\tau$-stage with $\ln (\tau)=2 e$ for some $e$, then if

$$
l(e, s)>\max \{l(e, t): t<s \text { and } t \text { is a } \tau \text {-stage }\},
$$

we say $s$ is a $\tau^{\wedge} 0$-stage; otherwise $s$ is a $\tau^{\wedge} 1$-stage.

(iii) If $s$ is a $\tau$-stage with $\ln (\tau)=2 e+1$ for some $e$, then if

$$
L(e, s)>\max \{L(e, t): t \text { is a } \tau \text {-stage and } t<s\}
$$

then $s$ is a $\tau^{\wedge} 0$-stage; otherwise $s$ is a $\tau^{\wedge} 1$-stage.

Followers of $P_{e}$ and their traces are only given guesses $\sigma$ with $\operatorname{lh}(\sigma)$ even and $\geqslant 2$. We say that $P_{e}$ requires attention if $P_{e}$ is not currently (declared) satisfied and one of the following options holds:

$$
\begin{aligned}
& q(e, s)>\max \{q(e, t): t<s\} \\
& \text { where } q(e, s)=\max \left\{x: \forall y<x\left(\hat{\Phi}_{e, s}\left(B_{s} ; y\right)=A_{s}(y)\right)\right\},
\end{aligned}
$$


and either

(a) $P_{e}$ is active via $x$, or

(b) $P_{e}$ is inactive but for some follower $x, q(e, s)>x$, or $P_{e}$ is inactive and has no follower.

Construction, Stage $s+1$.

Step 1. Define $\sigma_{s}$ as usual. Cancel and inactivate as usual for guesses $\tau{ }_{L} \sigma_{s}$.

Step 2. Find the least follower or trace $x$ (if any) targeted for $A$ for which there exist $\gamma$ and $\tau$ such that

(i) $x$ has guess $\gamma$,

(ii) $\operatorname{lh}(\tau)$ is even, say, $\operatorname{lh}(\tau)=2 e$,

(iii) $\tau^{\wedge} 0 \subset \gamma$ and $\tau^{\wedge} 0 \subset \sigma_{s}$,

(iv) $x$ is not yet $\tau^{\wedge} 0$-confirmed, and

(i) $l(e, s)>x$.

Declare $x$ as $\tau^{\wedge} 0$-confirmed and cancel all traces or followers targeted for $A$ or $B$ greater than $x$. Declare all $P_{j}$ affected by this as inactive.

Step 3. Find the least (if any) follower or trace $x$ targeted for $B$ for which there exist $\gamma$ and $\tau$ such that

(i) $x$ has guess $\gamma$,

(ii) $\operatorname{lh}(\tau)$ is odd, say, $\operatorname{lh}(\tau)=2 e+1$,

(iii) $\tau^{\wedge} 0 \subset \gamma$ and $\tau^{\wedge} 0 \subset \sigma_{s}$,

(iv) $x$ is not yet $\tau^{\wedge} 0$-confirmed, and

(v) $L(e, s)>x$.

Cancel and inactivate as in Step 2, above. Declare $x$ as $\tau^{\wedge} 0$-confirmed.

Step 4. Now find the least $e$ such that $P_{e}$ requires attention. Cancel all followers and traces with guess $\tau$ for $\tau \supset \sigma$ and $\tau \neq \sigma$ where $\sigma \subset \sigma_{s}$ and $\operatorname{lh}(\sigma)=2 e+2$. Inactivate appropriate $P_{j}$. Adopt the appropriate case below.

Case 1. (4.30) holds. Appoint $y=2 s+1$ as a follower of $P_{e}$ with guess $\sigma . P_{e}$ remains inactive. Declare $x$ and $x+1$ as both targeted for $A$, declare $x+1$ as a trace, and declare $x+1$ as targeted for $B$.

Case 2. (4.29) holds.

Subcase (i). (b) holds. Declare $P_{e}$ as active. Set $A_{s+1}=A_{s} \cup\{x+1\}$. Set $B_{s+1}=B_{s} \cup\{z: x+1 \leqslant z \leqslant 2 s\}$. Set $y(x)=2 s+1$ as $x$ 's new trace. Give $y(x)$ guess $\gamma$ where $\gamma$ is the guess of $x$. Declare $y(x)$ to be targeted for both $A$ and $B$. Cancel all followers and traces with guess $\eta$ for $\gamma \leqslant_{L} \eta$ and $\gamma \neq \eta$. Inactivate appropriate $P_{j}$.

Subcase (ii). (a) holds. Set $A_{s+1}=A_{s} \cup\{x, y(x)\}$ and $B_{s+1}=B_{s} \cup\{z: y(x) \leqslant z$ $\leqslant 2 s\}$. Declare $P_{e}$ as satisfied. Cancel, etc., as in subcase (i).

END OF CONSTRUCTION.

VerifiCation (SKETCH). Due to the evident similarities with (4.3) and (4.17), we sketch some of the details.

Let $\beta$ denote the leftmost path. We first verify the $R_{e}$. Let $s_{0}$ be a stage such that all the $P_{j}$ for $j<e$ cease acting and $\sigma \leqslant \sigma_{s}$ for all $s>s_{0}$ where $\sigma \subset \beta$ and $\ln (\sigma)=2 e+1$. 
Let $z$ be given. We wish to compute if $z \in W_{e}$ or not (from $A$ ). Find the least $\sigma$-stage $s_{1}>s_{0}$ with $l\left(e, s_{1}\right)>z$. We claim that, as in (4.17), if $s_{2}$ is the least $\sigma$-stage with $s_{2}>s_{1}$ and $A_{s_{2}}\left[s_{1}\right]=A\left[s_{1}\right]$, then

$$
z \in W_{e} \quad \text { iff } \quad z \in W_{e, s_{2}} .
$$

This follows by virtually the same argument as in (4.17). The crucial observation is that $y(x)$ may only enter $A$ at the same time as $x$. Briefly, to see (4.31) it suffices to observe that the only numbers $p$ left alive at stage $s_{2}$ with

$$
s_{1}<p<u \quad \text { where } u=u\left(\Phi_{e, s_{2}}\left(A_{s_{s}} ; z\right)\right)
$$

must be traces $y(x)$ and must be traces of some follower $x$ already present at stage $s_{1}$. Thus they can only enter $A$ at the same time as $x$. The rest of the argument mimics (4.17). Since we have transported the $N_{e}$ machinery virtually unchanged, the argument given in (4.3) will suffice for the $N_{e}$. Again we leave this to the reader.

We thus turn to the verification of the $P_{e}$. Let $s_{0}$ be an appropriate $\sigma$-stage as above (with $\operatorname{lh}(\sigma)=2 e+2$ and $\sigma \subset \beta)$. Now if $R_{e}$ fails to be met then $q(e, s) \rightarrow \infty$. Thus find a $\sigma$-stage $s_{1}>s_{0}$ where (4.30) holds. Then $P_{e}$ is given an uncancellable follower $x$. Find the least stage $s_{2}>s_{1}$ with $q\left(e, s_{2}\right)>x$. At such a stage we set $A_{s_{2}+1}=A_{s_{2}} \cup\{x+1\}$ and $B_{s_{2}+1}=B_{s_{2}} \cup\{x+1\}$. We also cancel all lower priority followers and traces.

The crucial observation is that the way we appoint followers and choice of $s_{0}$ ensures that

$$
B_{s_{2}+1}\left[\phi_{e}(x)\right]=B\left[\phi_{e}(x)\right]
$$

since $\phi_{e}(x)=u\left(\hat{\Phi}_{e, s_{1}}\left(B_{s_{1}} ; x\right)\right)=u\left(\hat{\Phi}_{e}(B ; x)\right)$. (Any number targeted for $B$ appointed after stage $s_{2}$ must exceed $s_{2}$ and there is no number left alive targeted for $B$ and $<s_{2}$ (except those that never get into $B$ covered by $s_{0}$ ).)

Thus, at the least stage $s_{3}>s_{2}$, with $q\left(e, s_{3}\right)>x,(4.29)$ (a) pertains to $x$ and we create a (permanent) disagreement

$$
\hat{\Phi}_{e}(B ; x)=0 \neq 1=A_{s_{3}+1}(x) .
$$

Finally, we verify that $A \equiv{ }_{\mathrm{T}} B$. To compute if $x \in A$ or not (from $B$ ) see if $x$ or $x-1$ is a follower or trace by stage $x$. If not and $x \notin A_{x}$ then $x \notin A$. If $x$ is a trace then $x \in A$ only if $x \in B$. If $x \in B$ find the stage $s$ where $x \in B_{s}-B_{s-1}$. Then $x \in A$ iff $x \in A_{s}$. If $x$ is a follower, $x \in A$ only if $x+1 \in B$ first. If $x+1 \in B$ find the stage $s$ where $x+1 \in B_{s}-B_{s-1}$. If $x$ is still alive at stage $s, x$ is given a trace $y(x)$. To then see if $x \in A$, find the stages (if any) where $y(x) \in B$. If none exists then $x \notin A$. If $y(x) \in B_{t}-B_{t-1}$ then $x \in A$ if $x \in A_{t}$. Hence $A \leqslant_{\mathrm{T}} B$. On the other hand $B \leqslant{ }_{\mathrm{T}} A$ by simple permitting.

We now give one surprising consequence of (4.28).

(4.33) Corollary. There exists an r.e. degree $\mathbf{a} \neq \mathbf{0}$ such that $\mathbf{a}$ contains infinitely many r.e. wtt-degrees and the structure of the r.e. wtt-degrees of degree $\mathbf{a}$ is a lattice with least and greatest elements. 
Proof. In [LS], Ladner and Sasso showed that if an r.e. degree is noncontiguous, then it contains infinitely many r.e. wtt-degrees. In [Fi] Paul Fischer constructed an r.e. nonzero wtt-degree $f$ such that the r.e. wtt-degrees below form a lattice. Now apply (4.28).

We remark that we do not know if (4.33) may be improved to say that the collection of all wtt-degrees of degree a form a lattice. (Note: By Exercise 3.5 of Chapter IX of Soare [So4], if a pair of r.e. wtt-degrees have an infimum in the wtt-degrees, the infimum is r.e.) We cannot use the technique above since for all r.e. wtt-degrees $\mathbf{f} \neq \mathbf{0}$ the wtt-degrees below $\mathbf{f}$ never form a lattice (cf. e.g. [Do3]).

We ask whether or not it is possible for the (r.e.) tt-degrees of an r.e. (wtt-) degree to also form a lattice. We also point out that degrees satisfying the properties of a of (4.32) would seem rare. We refer to [DS, Fi and Do3] for results on pairs without infimum in the r.e. wtt-degrees. We do not know what countable lattices can be embedded in this way, although Downey [Do3] has shown that they are not Boolean algebras.

As our final result we use the flexibility of the construction of (4.3) to answer a question of Odifreddi (personal communication) by showing that (strongly) tt-bottomed degrees do not necessarily contain minimal (r.e.) tt-degrees.

(4.34) THEOREM. There is a nonzero strongly contiguous strongly tt-bottomed degree a containing no sets of minimal (r.e.) tt-degree.

Proof (sketch). Build $A=\bigcup_{s} A_{s}$ and an auxiliary r.e. set $B=\bigcup_{s} B_{s}$ to satisfy

$$
\begin{aligned}
& N: B \leqslant{ }_{\mathrm{tt}} A . \\
& P_{e}: \bar{B} \neq W_{e} . \\
& N_{e}: \Gamma_{e}\left(\Phi_{e}(A)\right)=A \text { implies } A \leqslant{ }_{\mathrm{tt}} \Phi_{e}(A) \text { and } \Phi_{e}(A) \leqslant{ }_{\mathrm{wtt}} A . \\
& R_{e}: \Phi_{e}(B) \neq A .
\end{aligned}
$$

We meet the $N_{e}$ exactly as we did in (4.3) and (4.17). We actually ensure that $B \leqslant{ }_{\mathrm{m}} A$ by asking that a follower $x$ targeted for $B$ enters $B$ iff $x \in A$. By meeting the $R_{e}$ we ensure that $A{ }_{\mathrm{T}} B$. To meet these requirements we use a standard Friedberg-Muchnik procedure. Thus we first pick a follower $y$ targeted for $A$, cancelling all lower priority followers when we do so. We next wait till $\Phi_{e, s}\left(B_{s} ; y\right)=$ $A_{s}(y)$. At this stage we enumerate $y$ into $A$ and cancel all lower priority followers targeted for $B$. This clearly combines with our (4.3) strategy and creates a disagreement $\Phi_{e}\left(B_{0} ; y\right)=0 \neq 1=A(y)$.

We meet the $P_{e}$ by appointing followers $x$ and waiting till $x \in W_{e, s}$. Then add $x$ to both $A$ and $B$. The only interactions we must be careful with are those where if $x$ is a follower of $P_{e}$ that is later cancelled, we must still add $x$ to $B$ if ever $x$ enters $A$, because of the tt-condition we need to achieve. Nevertheless, it is really quite easy to blend the above with (4.3) and we leave this to the reader.

\section{REFERENCES}

[AS] K. Ambos-Spies, Contiguous r.e. degrees, Logic Colloquium '83, Lecture Notes in Math., vol. 1104, Springer-Verlag, Berlin and New York, 1984, pp. 1-37.

[ACJ] K. Ambos-Spies, S. B. Cooper, and C. Jockusch, Some relationships between Turing and weak truth table reducibilities (in preparation). 
[AF] K. Ambos-Spies and P. Fejer, Degree theoretic splitting properties of r.e. sets (to appear).

[AJSS] K. Ambos-Spies, C. Jockusch, R. Shore, and R. Soare, An algebraic decomposition of the recursively enumerable degrees and the coincidence of several degree classes with the promptly simple degrees, Trans. Amer. Math. Soc. 281 (1984), 109-128.

[Co] P. F. Cohen, Weak truth table reducibility and the pointwise ordering of the 1-1 recursive functions, Doctoral Dissertation, Univ. of Illinois, Urbana, Ill., 1975.

[Cp] S. B. Cooper, Minimal pairs and high recursively enumerable degrees, J. Symbolic Logic 39 (1974), $655-660$.

[De] J. C. E. Dekker, A theorem on hypersimple sets, Proc. Amer. Math. Soc. 5 (1954), 791-796.

[Dg1] A. N. Degtev, Hereditary sets and tabular reducibility, Algebra and Logic 11 (1972), 145-152.

[Dg2] _ Partially ordered sets of 1-degrees contained in a recursively enumerable m-degree, Algebra and Logic 15 (1976), 153-164.

[Do1] R. G. Downey, The degrees of r.e. sets without the universal splitting property, Trans. Amer. Math. Soc. 291 (1985), 337-351.

[Do2] _,$\Delta_{2}^{0}$ degrees and transfer theorems, Illinois J. Math. (to appear).

[Do3] __ Intervals and sublattices in the recursively enumerable wtt-degrees (submitted).

[DS] R. G. Downey and M. Stob, Structural interactions of the recursively enumerable $T$ - and $W$-degrees, Ann. Pure Appl. Logic 31 (1986), 205-236.

[Fi] P. Fischer, Pairs without infimum in the r.e. wtt-degrees, J. Symbolic Logic 51 (1986), 117-29.

[FR] R. Friedberg and H. Rogers, Reducibility and completeness for sets of integers, Z. Math. Logik Grundlag. Math. 5 (1959), 117-125.

[In] M. Ingrassia, P-genericity for recursively enumerable sets, Doctoral Dissertation, Univ. of Illinois, Urbana, Ill., 1981.

[Jo1] C. G. Jockusch, Reducibilities in recursive function theory, Doctoral Dissertation, M.I.T., Cambridge, Mass., 1966.

[Jo2] _ Relationships between reducibilities, Trans. Amer. Math. Soc. 142 (1969), 229-237.

[Jo3] _ Degrees in which the recursive sets are uniformly recursive, Canad. J. Math. 24 (1972), 1092-1099.

[Jo4] _ Genericity for recursively enumerable sets, Recursion Theory Week (H. Ebbinghaus, G. Müller and G. Sacks, eds.), Lecture Notes in Math., vol. 1141, Springer-Verlag, New York, 1985, pp. 203-232.

[Ko1] G. N. Kobzev, On btt-reducibility. II, Algebra and Logic 12 (1973), 443-444.

[Ko2] _ Relationships between recursively enumerable tt-and w-degrees, Bull. Akad. Sci. Georgian SSR 84 (1976), 585-587.

[Ko3] _ On the tt-degrees of r.e. T-degrees, Mat. Sb. 106 (1978), 507-514.

[La1] A. H. Lachlan, Lower bounds for pairs of recursively enumerable degrees, Proc. London Math. Soc. 16 (1966), 537-568.

[La2] _ Recursively enumerable many-one degrees, Algebra and Logic 11 (1972), 186-202.

[La3] _ wtt-complete sets are not necessarily tt-complete, Proc. Amer. Math. Soc. 48 (1975), 429-434.

[La4] __ B Bounding minimal pairs, J. Symbolic Logic 44 (1979), 626-642.

[Ld] R. Ladner, A completely mitotic nonrecursive r.e. degree, Trans. Amer. Math. Soc. 184 (1973), 479-507.

[LS] R. Ladner and L. P. Sasso, The weak truth table degrees of recursively enumerable sets, Ann. Math. Logic 8 (1975), 429-448.

[LR] M. Lerman and J. Remmel, The universal splitting property. II, J. Symbolic Logic 49 (1984), 137-150.

[Od1] P. Odifreddi, Strong reducibilities, Bull. Amer. Math. Soc. (N.S.) 4 (1984), 37-86.

[Od2] ___ Classical recursion theory (to appear).

[Po] D. Posner, The upper semilattice of degrees $\leqslant \mathbf{0}^{\prime}$ is complemented, J. Symbolic Logic 46 (1981), 705-713.

[Ps] E. Post, Recursively enumerable sets and their decision problems, Bull. Amer. Math. Soc. 50 (1944), 284-316.

[Ro] R. W. Robinson, Interpolation and embedding in the recursively enumerable degrees, Ann. of Math. (2) 93 (1971), 285-314.

[Rg] H. Rogers, Theory of recursive functions and effective computability, McGraw-Hill, New York, 1967.

[So1] R. I. Soare, Recursion theory and Dedekind cuts, Trans. Amer. Math. Soc. 140 (1969), 271-294. 
[So2] $545-563$. , Computational complexity, speedable and levelable sets, J. Symbolic Logic 42 (1977), [So3] Tree arguments in recursion theory and the $\mathbf{0}^{\prime \prime \prime}$ priority method, Recursion Theory (A. Nerode and R. Shore, eds.), Proc. Sympos. Pure Math., vol. 42, Amer. Math. Soc., Providence, R.I., 1985, pp. 53-106.

[So4] __ Recursively enumerable sets and degrees, Springer-Verlag Omega Series (to appear).

[St] M. Stob, wtt-degrees and T-degrees of r.e. sets, J. Symbolic Logic 48 (1983), 921-930.

[Ya] C. E. M. Yates, Degrees of index sets. II, Trans. Amer. Math. Soc. 135 (1969), 249-266.

Department of Mathematics, Victoria University of Wellington, Private Bag, Wellington, New Zealand

Department of Mathematics, University of Illinois, 1409 W. Green St., Urbana, Illinois 61801 